Portland State University

PDXScholar

Civil and Environmental Engineering Faculty

Publications and Presentations

$1-1-1990$

\title{
Residual Circulation in Shallow Estuaries 2. Weakly Stratified and Partially Mixed, Narrow Estuaries
}

\author{
David A. Jay \\ Portland State University \\ J. Dungan Smith \\ University of Washington - Seattle Campus
}

Follow this and additional works at: https://pdxscholar.library.pdx.edu/cengin_fac

Part of the Civil and Environmental Engineering Commons

Let us know how access to this document benefits you.

\section{Citation Details}

Jay, D. A., and J. D. Smith (1990), Residual Circulation in Shallow Estuaries 2. Weakly Stratified and Partially Mixed, Narrow Estuaries, J. Geophys. Res., 95(C1), 733â€"748.

This Article is brought to you for free and open access. It has been accepted for inclusion in Civil and Environmental Engineering Faculty Publications and Presentations by an authorized administrator of PDXScholar. Please contact us if we can make this document more accessible: pdxscholar@pdx.edu. 


\title{
Residual Circulation in Shallow Estuaries 2. Weakly Stratified and Partially Mixed, Narrow Estuaries
}

\author{
David A. Jay and J. Dungan Smith
}

Geophysics Program, University of Washington, Seattle

\begin{abstract}
Long-wave theory is used herein to analyze circulation in weakly stratified and partially mixed estuaries. Unlike the highly stratified systems considered in part 1 , the flows considered here have only a minimal tidal-frequency internal wave component. These estuaries may therefore be modeled as the sum of weakly interacting barotropic and baroclinic modes. The dominant factors driving the residual flow are finite amplitude barotropic effects in weakly stratified estuaries and a combination of barotropic effects and steady horizontal density gradient forcing in partially mixed estuaries. The dominant vertical exchange mechanism in the weakly stratified case is bottom boundary-induced turbulence, and that in partially mixed estuaries is believed to be random internal wave interactions. A model of the Columbia River Estuary under weakly stratified conditions accurately predicts the observed residual velocity and salinity fields, and the tidal amplitude at which a transition to a highly stratified state takes place. The partially mixed state is unstable in the Columbia River Estuary because the tides and buoyancy input are both too strong.
\end{abstract}

\section{Introduction: Weakly Stratified and Partially Mixed Estuarine Flows}

The purpose of this article is to investigate the character of estuarine circulation in weakly stratified and partially mixed estuaries, using the long-wave perturbation methods already employed by Jay and Smith [this issue] (hereinafter referred to as part 1). Observations suggest and our analysis will confirm that tidal-frequency internal oscillations do not, in contrast to the situation considered in part 1 , play an important role in these systems. Previous investigations of this class of estuary have focussed either on nonlinear residual flow generation mechanisms associated with a finite amplitude barotropic tide or on the residual generated by a mean density gradient. In the latter case, the tide is treated only parametrically. We demonstrate herein that these two approaches are components of a more general analysis that eliminates the need for parametric representation of the tide, and that the nature of the dominant vertical exchange mechanism strongly influences the character of both tidal and residual flows. Barotropic advection of salt by both the tidal and residual flows plays a much larger role than the density-driven circulation in maintaining the salt balance in weakly stratified estuaries. Baroclinic advection of salt becomes increasingly important in partially mixed estuaries as mixing weakens and stratification increases.

Weak stratification and strong tidal advection of a nearly uniform horizontal density field occur throughout most of the tidal month during the low-flow season in the Columbia River Estuary. Strong stratification is observed during and after neap tides, but once the system has shifted from highly stratified to weakly stratified after the neap, there is little variation in the density field over

Copyright 1990 by the American Geophysical Union. a large part of the tidal month (Figure 1 of part 1). Velocity and density profiles and density sections (e.g., Figures 4 and 8 , part 1) strongly suggest that bottom boundaryinduced turbulent mixing is able to extend throughout most of the flow. It is absent only near the free surface, where the shear nearly vanishes (e.g., Figure 8 of part 1). Thus a weakly stratified estuary can to a first approximation be treated as a stratified boundary layer. Furthermore, because bottom boundary-induced turbulence encompasses a large part of the flow, it is the dominant process causing the vertical exchange of salt and fresh water on the flood in weakly stratified estuaries. Mixing on the flood is of particular interest, because observations (part 1) show and model results confirm that the various circulation types are most distinct on the flood. The flood is, therefore, the decisive part of the tidal cycle governing the form of the residual flow. It is best not to refer to the "weakly stratified" estuaries discussed as herein as "well mixed," because the latter implies that stratification is absent, which is not the case. Although it is weak, stratification is necessary to the maintenance of salinity intrusion [ Jay, 1989] and its intensification during periods of weak tidal forcing causes the spring-neap transition. This transition (section 3 ) occurs around the time of the neap tides (Figure 1 of part 1), when the tidal range decreases to the point that the gradient Richardson number $R i_{g}$ exceeds a critical value throughout the interior of the flow on flood. Mixing decreases drastically, and the flow becomes highly stratified.

The transitions between highly and weakly stratified regimes is rapid in the Columbia River Estuary (a few days or one flushing time), and the intermediate, partially mixed regime is only transient. The analysis for the weakly stratified state is extended in section 4 to the partially mixed state to investigate this transiency. The stratification in partially mixed estuaries is sufficient to extinguish boundary-induced turbulent mixing in the interior. Kelvin-Helmholtz instabilities are also largely absent, because the stratification is insufficient to sustain a tidal-frequency internal oscillation, so tidal shear in the 
interior is relatively weak. In the absence of stronger mechanisms, random interaction and consequent breaking of internal waves are hypothesized to be the dominant source of vertical exchange of salt and fresh water. The identity of the dominant mixing mechanism is less important, however, than the fact that vertical mixing in the partially mixed state is weak relative to that in the weakly and highly stratified cases. Model results show that the partially mixed condition is not stable in the Columbia River Estuary because the river flow is always sufficient to return the system to a highly stratified state whenever turbulent mixing cannot be maintained in the interior on flood. Conversely, tidal forcing is strong enough that the system becomes weakly stratified whenever internal mixing renders a two-layer tidal advance impossible.

The strategy used in this analysis is to develop separate perturbation expansions for the barotropic and baroclinic modes, beginning with the simpler barotropic flow (sections 2 and 3 ). Because large, tidal-frequency internal oscillations are absent, the $O(1)$ barotropic mode depends on the baroclinic mode only insofar as the eddy diffusivity is affected by the stratification. In the absence of an $O(1)$ baroclinic mode, the mean horizontal density gradient is taken to be spatially and temporally uniform. Tidal advection of the assumed uniform density field then leads to small vertical and horizontal density variations.

The barotropic analysis of section 2 is formally similar to that used by Ianniello $[1977 a, b]$ to investigate nonlinear generation of residual flow by finite amplitude effects in a channel of uniform width and depth. This method is extended to include the salt conservation equation herein. Ianniello demonstrated that a vertically integrated analysis of barotropically forced residual flow was inadequate to describe processes as basic as the direction of residual particle transport near the bed, because substantial shear and flow reversal may develop in the barotropic residual current, just as in a steady density-driven flow. This conclusion is considerable amplified when barotropic and baroclinic effects are combined herein, because damping by stratification of vertical turbulent exchange allows the shear to be much greater.

From an internal long wave point of view, the flow in weakly stratified and partially mixed estuaries is degenerate, because of the absence of an $O(1)$ internal wave motion. Our baroclinic analysis thus resembles that first developed by Hansen and Rattray [1965] for the "central regime" and used in numerous subsequent studies. In the central regime theory, the assumption of a constant (in time and space) along-channel salinity gradient throughout the central part of an estuary (along-channel similarity), use of a constant vertical eddy diffusivity and representation of tidal salt advection in terms of a horizontal mixing coefficient allow reduction of the steady, coupled nonlinear momentum, mass, and salt conservation equations to a linear system in one independent variable (the vertical coordinate). Our analysis eliminates the arbitrary constants in central regime theory by representing tidal advection explicitly and appropriately specifying the vertical eddy diffusivity. Moreover, it makes possible for the first time characterization of both barotropic and baroclinic effects in estuarine circulation without recourse to numerical models.

\section{Development and Solution of THE Governing Equations}

The geometry assumed for the weakly stratified is the same as was assumed in the two-layer case 1) - the channel is narrow and of uniform width depth. As before, the flow is assumed to be essent two-dimensional in the along-channel and vertical di tions. This excludes systems in which the salt balance maintained by horizontally extensive (in two dimensions) chaotic eddy dispersion processes. The basic solution termined for the constant width and depth case is modified to account for the along-channel decrease in transport caused by salt water flowing out over the tensive tidal flats in the lower Columbia River Est Because the salt balance in the weakly stratified regin is maintained primarily by the tidal flow, the principal effect of this along-channel decrease in tidal transport is a small, along-channel compression in the spacing of the density contours. This effect was unimportant in part 1 , because the stratification confined the salt to the deeper channels.

Topographic effects are excluded from the present analysis, just as in part 1 . This assumes that the spring-neap transition, like the neap-spring transition, is caused global change in mixing processes throughout the ary, rather than by a topographically mediated proce a particular point. The limited role of specific topographic features under weakly stratified conditions is supported by the small along-channel changes in stratification in Figures $4 a-4 c$ of part 1 as the flow passes over a series of sills and holes. It is also hard to imagine how the spring-neap transition could be caused by an isolated deep hole in the channel bottom, because vigorous mixing would occur on the flood just upstream of the hole. A model of salinity intrusion in a weakly stratified channel of constant width and depth is therefore a reasonable first step toward a more complete analysis of a channel with variable width and depth. The success of Ianniello's $[1979,1981]$ analysis of barotropic flow over topography suggests that the present analysis can be extended to include so long as the flow does not become fully three-dimensional.

The analysis follows from the equations for conservation of vertical and horizontal momentum, mass and salt. The hydrostatic assumption is made, because the horizontal scale is, as in all long waves, very large relative to the vertical. No horizontal diffusion term is included in the mass conservation equation, because tidal advection of salt is explicitly represented and horizontal turbulent diffusion may be assumed small relative to horizontal advection and vertical turbulent mixing. It is convenient to divide the flow into weakly interacting barotropic and baroclinic modes. This is possible because the baroclinic flow is only $O(\epsilon)$ and none of the bilinear terms in the momentum and salt conservation equations involving the baroclinic variables appear in the $O(1)$ tidal frequency perturbation equations to follow. The velocity may be written

$$
\begin{aligned}
\widehat{U} & =\widehat{U}_{i}+\widehat{U}_{t} \\
\widehat{W} & =\widehat{W}_{i}+\widehat{W}_{t}
\end{aligned}
$$

where $\widehat{U}$ is horizontal velocity and $\widehat{W}$ is vertical velocity. 
The subscript " $i$ " refers to the baroclinic (internal) mode and " $t$ " refers to the barotropic mode.

\section{The Barotropic Mode}

The governing equations for the barotropic mode are a subset of the full equations

$$
\begin{aligned}
& \frac{\partial \widehat{U}_{t}}{\partial \widehat{t}}+\widehat{U}_{t} \frac{\partial \widehat{U}_{t}}{\partial \widehat{x}}+\widehat{W}_{t} \frac{\partial \widehat{U}_{t}}{\partial \widehat{z}}=-g \frac{\partial \widehat{h}_{t}}{\partial \widehat{x}} \\
&+\frac{\partial}{\partial \widehat{z}}\left(K_{m} \frac{\partial \widehat{U}_{t}}{\partial \widehat{z}}\right) \\
& \frac{\partial \widehat{U}_{t}}{\partial \widehat{x}}+\frac{\partial \widehat{W}_{t}}{\partial \widehat{z}}=0 \\
& \frac{\partial}{\partial \widehat{x}} \int_{-H}^{\widehat{h}} \widehat{U}_{t} d z+\frac{\partial \widehat{h}_{t}}{\partial \widehat{t}}=0
\end{aligned}
$$

where

\section{horizontal distance;}

vertical distance;

time;

surface elevation;

$=9.8 \mathrm{~m} \mathrm{~s}^{-2}$

$K_{m} \quad$ vertical eddy diffusivity;

$H$ depth of flow.

These are the equations used by Ianniello $[(1977 a, b]$ in his analysis of barotropic residual circulation in a uniform channel in the absence of baroclinic forcing. The perturbation analysis and solution of these equations herein is identical to Ianniello's, except for minor differences in scaling and notation and a different treatment of the eddy diffusivity. The equations are scaled and nondimensionalized under the assumption that in a channel of uniform cross section, the along-channel length scale is set by the tidal wave length:

$$
\begin{aligned}
\widehat{U}_{t}=\mathcal{U}_{b} U_{t} & \widehat{x}=\lambda x \\
\widehat{W}_{t}=\mathcal{U}_{b} \frac{H}{\lambda} W_{t} & \widehat{z}=H z \\
\widehat{h}=\zeta h & \widehat{t}=\frac{t}{\omega}
\end{aligned}
$$

where

$u_{b}=\zeta / H c \sim 1 \mathrm{~m} \mathrm{~s}^{-1}$

$\lambda$ barotropic tidal wavelength;

$H \simeq 16 \mathrm{~m}$;

$\zeta$ tidal amplitude, equal to $1 \mathrm{~m}$;

$\omega$ tidal frequency, equal to $1.4 \times 10^{-4} \mathrm{~s}^{-1}$.

Variables without carets are nondimensional. This scaling assumes that the tidal flow is unaffected by the variations in the baroclinic flow on shorter length scales. The perturbation parameter is $\epsilon_{b}=\zeta / H$, the ratio of tidal amplitude to depth. As discussed in part $1, \epsilon_{b}$ is a Froude number that indicates the extent of barotropic nonlinearity in the channel. The perturbation expansion yields terms of various frequencies and order. The various terms in each order and each frequency must balance one another. It is assumed that only the tidal frequency flow is $O(1)$.
This assumption is used because the nonlinear terms that drive the overtide and residual flows scale as $O\left(\epsilon_{b}\right)$ in the analysis that follows. It is, moreover, commonly observed that both the residual and overtide flows are small relative to the basic tidal flow near estuary mouths. Equations at residual and overtide frequencies appear at $O\left(\epsilon_{b}\right)$; of these, only the residual flow equations will be discussed here.

\section{The Barotropic Tidal Flow}

The $O(1)$ equations of motion and continuity that result from the perturbation expansion of the nondimensional versions of $(2 a)$ and $(2 b)$ are:

$$
\begin{gathered}
\frac{\partial^{1} U_{t}^{0}}{\partial t}-\frac{\partial}{\partial z}\left(\frac{K_{m}}{\omega H^{2}} \frac{\partial^{1} U_{t}^{0}}{\partial z}\right)=\frac{\partial^{1} h_{t}^{0}}{\partial x} \\
\frac{\partial^{1} U_{t}^{0}}{\partial x}+\frac{\partial^{1} W_{t}^{0}}{\partial z}=0 \\
\frac{\partial}{\partial x} \int_{-1}^{0}{ }^{1} U_{t}^{0} d z+\frac{\partial^{1} h_{t}^{0}}{\partial t}=0
\end{gathered}
$$

The left superscript indicates the frequency of the term (" 0 " for mean flow, " 1 " for tidal flow, and " 2 " for overtides), and the right superscript indicates the order in the perturbation expansion (" 0 " for highest order and " 1 " for $\left.O\left(\epsilon_{b}\right)\right)$. Nondimensional, tidal-frequency mass conservation (equation (4c)) differs from $(2 c)$ in that the upper bound of the integral is the mean position of the free surface. The time average of the remainder of the integral (from the mean position of the free surface to its instantaneous position) is in general, nonzero. This correlation of fluid particle displacement and velocity is the Stokes drift and represents the mass carried forward in the crests of the waves but not carried backward in the troughs. It appears below in the $O\left(\epsilon_{b}\right)$ continuity equation.

Solution of $(4 a)$ to $(4 c)$ requires an assumption concerning the form of the eddy diffusivity $K_{m}$. There are three basic options: (1) numerical integration of using an eddy diffusivity profile determined by a simple turbulence closure with a stratification correction, (2) use of a piecewise continuous eddy diffusivity profile that allows analytical integration of ( $4 a)$, if appropriate matching conditions are specified, and (3) assumption of a constant (in the vertical) eddy diffusivity with a modified bottom boundary condition to allow a small slip velocity, so that the analytical solution yields the same velocity as a logarithmic profile at a matching depth near the bottom. The first alternative would be the method of choice in a study where detailed predictions for a particular estuarine topography were required. The purpose of this study is not, however, to calculate site-specific flow fields, but to obtain a qualitative understanding of the important flow processes through direct, analytical solution of the governing equations. Ianniello $[1977 b, 1979,1981]$ has shown by comparison of flow fields calculated with and without topography that convective accelerations associated with topographic effects, and different parameterizations of $K_{m}$ cause changes in the velocity profile of similar order in a neutrally stratified flow. In a stratified flow, complex interactions between topography, shear and stratification 
can be expected. It seems logical, therefore, to exclude topography and begin with the simplest possible form of $K_{m}$.

This leaves the second and third alternatives. Both of these allow analytical solution of $(4 a)$ to $(4 c)$ and are superior to use of a constant eddy diffusivity with a noslip boundary condition, because a proper boundary shear stress and approximately correct mixing in the interior may be simultaneously achieved. A stratification correction based on the results of the baroclinic mode analysis may be added to either of these two alternatives by iteration between the barotropic and baroclinic mode calculations. Given the purpose of this study, either alternative would be acceptable, but the third has been adopted, because it leads to simpler algebra.

The assumption of a constant eddy diffusivity allows a separation of variables; the tidal surface elevation and horizontal velocity may be represented:

$$
\begin{aligned}
{ }^{1} h_{t}^{0}(x, t) & =\operatorname{Re}\left(M(x) e^{i t}\right) \\
{ }^{1} U_{t}^{0}(x, z, t) & =\operatorname{Re}\left(i M^{\prime}(x) P(z) e^{i t}\right)
\end{aligned}
$$

where a prime indicates a spatial derivative and $\operatorname{Re}(f)$ is the real part of the complex function $f$. Conservation of momentum (equation (4a)) and mass (equation (4c)) become:

$$
\begin{gathered}
P^{\prime \prime}-2 i m^{2} P=-2 i m^{2} \\
M^{\prime \prime}+\left(1+\left.\frac{i}{2 m^{2}} P^{\prime}\right|_{-1}\right)^{-1} M=0
\end{gathered}
$$

where $m$ is the nondimensional depth, equal to $\left[\omega H^{2} /\right.$ $(2 K)]^{1 / 2}$. The boundary conditions for $(6 a)$ are a slip condition at the bed (at $z=-1$ ) and an unstressed free surface (at $z=0$ ):

$$
\begin{aligned}
& \left.{ }^{1} U_{t}^{0}\right|_{-1}={ }^{1} V_{s}^{0} \\
& \left.\frac{\partial^{1} U_{t}^{0}}{\partial z}\right|_{0}=0
\end{aligned}
$$

Those for $(6 b)$ require specification of the tidal amplitude at the entrance and far upriver:

$$
\begin{array}{lll}
{ }^{1} h^{0}=H_{0} & \text { at } & x=0 \\
{ }^{1} h^{0}=0 & \text { at } & x=\infty
\end{array}
$$

The solutions to $(6 a)-(6 c)$ with conditions $(7 a)$ to $(7 d)$ are

$$
\begin{aligned}
P(z) & =1+A \cosh (1+i) m z \\
A & =\frac{{ }^{1} V_{s}^{0}-1}{\cosh (1+i) m} \\
M(x) & =H_{0} e^{-i q x} \\
q & =\left(1+\left.\frac{i}{2 m^{2}} P^{\prime}\right|_{-1}\right)^{-1 / 2}
\end{aligned}
$$

where $q$ is complex wave number. The condition at infinity (equation $(7 d)$ ) allows only an incident wave, which damps as it propagates; there is no reflected wave. Ianniello's $[1977 a, b]$ solution for constant $K_{v}$ is recovered, if the slip velocity at the boundary ${ }^{1} V_{S}^{0}$ is set to zero. The vertical velocity ${ }^{1} W_{t}^{0}$ may then be found from $(4 b)$ and the first integral of $(6 a)$. Use of the boundary condition

$$
\left.{ }^{1} W_{t}^{0}\right|_{-1}=0
$$

determines ${ }^{1} W_{t}^{0}$ as

$$
{ }^{1} W_{t}^{0}=-\operatorname{Re}\left[i M^{\prime \prime}\left(z+1-\left.\frac{i}{2 m^{2}} P^{\prime}\right|_{-1} ^{z}\right) e^{i t}\right]
$$

\section{The Barotropic Residual Flow}

The $O\left(\epsilon_{b}\right)$ residual momentum and continuity equations that result from the perturbation expansion of $(2 a)$ and $(2 b)$ are

$$
\begin{gathered}
\left(\overline{{ }^{1} U_{t}^{0} \frac{\partial^{1} U_{t}^{0}}{\partial x}}+\overline{{ }^{1} W_{t}^{0} \frac{\partial^{1} U_{t}^{0}}{\partial z}}\right)= \\
-\frac{\partial^{0} h_{t}^{1}}{\partial x}+\frac{K}{\omega H^{2}} \frac{\partial^{2}{ }^{0} U_{t}^{1}}{\partial z^{2}} \\
\frac{\partial^{0} U_{t}^{1}}{\partial x}+\frac{\partial^{0} W_{t}^{1}}{\partial z}=0 \\
\frac{\partial}{\partial x} \int_{-1}^{0}{ }^{0} U_{t}^{1} d z+\frac{\partial Q_{S}}{\partial x}=0 \\
\int_{-1}^{0}{ }^{0} U_{t}^{1} d z+Q_{S}=Q_{R}
\end{gathered}
$$

where

$$
Q_{S}=\overline{\left.{ }^{1} U_{t}^{0}\right|_{0} ^{1} h_{t}^{0}}
$$

is Stokes drift, $Q_{R}$ is nondimensional river flow, and $\bar{f}$ is tidal cycle average of $f$. Conservation of mass $(10 c)$ indicates that the divergence of the horizontal transport is balanced by the divergence of the Stokes drift. The Stokes drift arises physically from the forward mass transport caused by the finite amplitude of the tidal wave. This nonlinearity scales with the perturbation parameter $\epsilon_{b}$. Mathematically, the Stokes drift term comes from a time average of a Taylor series representation (truncated after two terms) of the portion of the transport integral (equation $(2 c)$ ) that lies between the mean position of the free surface at $\mathrm{z}=0$ and the actual free surface. There is a corresponding term at the frequency of the first overtide. The validity of this truncation is indicated by the fact that the next term in the expansion vanishes so long as the free surface is unstressed. The result (equation $(10 d)$ ) of integration of mass conservation (equation (10c)) from $x$ to infinity under the assumption that all river flow enters at the upstream end of the system. The validity of $(10 a)$ is subject to the demonstration below that no $O(1)$ baroclinic flow exists to add additional driving terms. 
Evaluation of the time averages in the Stokes drift $Q_{S}$ and the nonlinear driving terms in $(10 a)$ is carried out using (equation (13)) of part 1, (5), $(6 b),(8 c)$, and (9), and their complex conjugates:

$$
Q_{S}=\operatorname{Re}\left[\left.\frac{i}{2} M^{\prime \prime *} M^{\prime}\left(1-\left.\frac{i}{2 m^{2}} P^{\prime *}\right|_{-1}\right) P\right|_{0}\right]
$$

$$
\begin{aligned}
\overline{1} U_{t}^{0} \frac{\partial^{1} U^{0}}{\partial x} & \overline{1} W_{t}^{0} \frac{\partial^{1} U_{t}^{0}}{\partial z} \\
= & \operatorname{Re}\left\{M ^ { \prime \prime * } M ^ { \prime } \left[|P|^{2}\right.\right. \\
& \left.\left.-P^{\prime}\left(z+1+\left.\frac{i}{2 m^{2}} P^{\prime *}\right|_{-1} ^{z}\right)\right]\right\}
\end{aligned}
$$

$$
\begin{aligned}
& U_{s}=\overline{\int{ }^{1} U_{t}^{0} d t \frac{\partial{ }^{1} U_{t}^{0}}{\partial x}}+\overline{\int{ }^{1} W_{t}^{0} d t \frac{\partial{ }^{1} U_{t}^{0}}{\partial z}} \\
& =\operatorname{Re}\left\{\frac { - i } { 2 } M ^ { \prime \prime * } M ^ { \prime } \left[|P|^{2}\right.\right. \\
& \left.\left.+P^{\prime}\left(z+1+\left.\frac{i}{2 m^{2}} P^{\prime *}\right|_{-1} ^{z}\right)\right]\right\}
\end{aligned}
$$

The vertical integral of $U_{S}$ is the Stokes drift equation $(11 a))$. This completes the determination of the barotropic velocity field.

\section{Character of the Baroclinic Flow}

The highly stratified flow analyzed in part 1 was strongly baroclinic - the $O(1)$ equations describe a salt wedge advance and retreat driven in part by a barotropic tide. Observational evidence suggests, however, that a baroclinic flow of comparable strength does not exist in the weakly stratified case. The dominant feature is barotropic tidal advection of the density field. The relative success of the Hansen and Rattray [1965] and other similar models that do not represent explicitly the tidal flow also argues that $O(1)$ baroclinic motions do not exist in this case. One physical reason for the nonexistence of this $O(1)$ mode is the absence (at $O(1))$ of a gravitational restoring force.

The weakness of the gravitational restoring force can be demonstrated by scaling and nondimensionalizing the relevant internal wave equation. Manipulation of the equations expressing the conservation of momentum, mass, and salt to obtain this wave equation is the same as that normally used for internal waves in a continuously stratified flow field [e.g., Turner, 1973] except that vertical diffusion, tidal advection of the density field, a horizontal density gradient, and barotropic forcing must included. This equation may then be expressed in terms of a regular perturbation expansion. The result is an $O(1)$ equation for horizontal velocity $U_{i}$ or vertical velocity $W_{i}$ containing a stress divergence term that is fourth order in $z$. The gravitational restoring force term proportional to $\mathcal{N}^{2}=-g / \rho_{o}(\partial \bar{\rho} / \partial z)$ does not, however, appear at lowest order, because the stratification is weak. Jay [1987] has analyzed the inviscid analog (neglecting the stress divergence) of the above $O(1)$ wave equation and shown that because of the lack of a gravitational restoring force, it lacks physically meaningful wave solutions.

The perturbation parameter used in the above expansion remains to be specified. Its identity explains in part the relationship of the present analysis to that in part 1 for the highly stratified state. Because the present perturbation analysis is designed to investigate the possible existence of $O(1)$ tidal-frequency internal wave motion, the perturbation parameter $\epsilon_{i}$ should measure the tendency for wavelike motion to occur. It is conveniently nondimensionalized by a measure of the strength of the steady horizontal density gradient. The horizontal density forcing in the internal wave equation mentioned in the previous paragraph is stronger than the vertical buoyant forcing by the ratio
The solution for ${ }^{0} U_{t}^{1}((12)$ and $(13 a, b))$ are exactly as given by Ianniello $[1977 a, b]$ for ${ }^{0} V_{s t}^{1}={ }^{1} V_{s}^{0}=0$. Finally, since the salinity field is advected by the total Lagrangian velocity, it is useful to determine the Stokes velocity $U_{S}$ as defined by Longuet-Higgins [1969], such that the total Lagrangian velocity ${ }^{0} U_{l}^{1}$ is the sum of ${ }^{0} U_{t}^{1}$ and $U_{S}$ : 


$$
\frac{1}{\epsilon_{i}^{2}}=\frac{\left[\mathcal{M}^{2} H /\left(\omega^{2} L_{s}\right)\right]}{\left[\mathcal{N}^{2} H^{2} /\left(\omega^{2} L_{s}^{2}\right)\right]} \cong \frac{\Delta \rho_{h}}{\Delta \rho_{v}} \sim 10 \text { to } 50
$$

where

$$
\mathcal{M}^{2}=\left(-g / \rho_{o}\right)(\partial \bar{\rho} / \partial x)
$$

$L_{s} \quad$ salinity intrusion length;

$\rho_{o}$ reference density;

$$
\begin{aligned}
& \Delta \rho_{v} \quad \text { scale top-to-bottom vertical density } \\
& \text { difference; }
\end{aligned}
$$

$\Delta \rho_{h} \quad$ scale horizontal density difference along the estuary.

The inverse root of this ratio $\epsilon_{i}=\left(\Delta \rho_{v} / \Delta \rho_{h}\right)^{1 / 2}$ is the desired perturbation expansion parameter. So long as $\epsilon_{i}$ is small, no $O(1)$ tidal frequency internal wave motion will occur, because the gravitational restoring force is weak relative to the horizontal density forcing. The relationship of $\epsilon_{i}$ to the perturbation parameter for the two-layer case can be understood by defining a velocity scale for the putative $O(1)$ baroclinic mode, as driven by the horizontal density gradient. The appropriate baroclinic velocity scale $\mathcal{U}_{i}$ is then

$$
\mathcal{U}_{i}=\left(\frac{\Delta \rho_{h}}{\rho_{0}} g H\right)^{1 / 2}
$$

and the internal scaling Froude number $F_{i}$ is the inverse of $\epsilon_{i}$ :

$$
F_{i}=\frac{\mathcal{U}_{i}}{g^{\prime} H}=\left(\frac{\Delta \rho_{h}}{\Delta \rho_{v}}\right)^{1 / 2}>>1
$$

where

$$
\hat{g}^{\prime}=g\left(\Delta \rho_{v} / \rho_{o}\right)
$$

The expansion parameter for the two-layer case (part 1) was an internal scaling Froude number and is therefore equivalent to the inverse of $\epsilon_{i}$. The analyses of estuarine flow herein and in part 1 thus define two asymptotic cases - strongly and weakly stratified - that suffice to describe the circulation during most of the tidal month in the Columbia River Estuary. The perturbation parameter in each case measures the tendency of the system to undergo a transition to the other state. In the two-layer case, the internal tidal motion was largely responsible for the generation of residual flow. In the weakly stratified flow discussed here, the barotropic tidal motion will turn out to be dominant.

That the internal Froude number is much greater than 1 suggests a second physical reason why tidal-frequency internal oscillations are not observed in weakly stratified estuaries: if they existed, then the interfacial mixing induced by their presence would cause destruction (by internal mixing) of the stratification that supported these oscillations. To prove this, it suffices to show that the vertical mixing scale length $\Delta i$ in the presence of such a hypothetical internal oscillation is greater than $H$, the depth of the flow. Proceeding as in (15b) of part 1 , and taking $\mathcal{U}_{i}$ for an $O(1)$ internal oscillation in the presence of strong horizontal density forcing as per (16a),

$$
\Delta \widehat{i}=\frac{R i_{g} \mathcal{U}_{i}^{2}}{g^{\prime}}>R i_{g} \frac{\Delta \rho_{h}}{\Delta \rho_{v}} H>H
$$

Tidal-frequency, internal oscillations are then inconsistent with the weakly stratified state. Stable, wavelike
$O(1)$ baroclinic motion at tidal frequencies is possible only when $\Delta \rho_{v}$ dominates over $\Delta \rho_{h}$, but this is precisely the two-layer case analyzed in part 1 . Thus, as suggested by observations (e.g., Figures $4 a-4 c$ of part 1), the $O(1)$ baroclinic, tidal-frequency mode may be neglected in the analysis of weakly stratified estuarine flow that follows.

\section{Steady Flow Driven by a Horizontal Density Gradient}

The results of the previous section justify the assumption that baroclinic flows in weakly stratified estuaries are weak. The absence of $O(1)$ tidal-frequency, baroclinic velocity allows a greatly simplified analysis of the baroclinic mode, because zero-frequency nonlinear interactions between the barotropic and baroclinic modes are not possible before $O\left(\epsilon^{2}\right)$. The tidal advection of the salinity field is, however, a very prominent feature in the observations, and the absence of an $O(1)$ baroclinic velocity does not exclude barotropic advection of the salinity field from the $O(1)$ analysis. The first problem is therefore to determine this advection under the assumption that the horizontal density forcing is nearly constant. The salinity and salinity gradient are assumed to be of the form

$$
\begin{aligned}
\widehat{S}(x, z, t)= & S_{0}\left[{ }^{1} S^{0}(x, t)+{ }^{0} S^{0}(x)\right. \\
& +\epsilon_{i}\left({ }^{0} S^{1}(x, z)+{ }^{1} S^{1}(x, z, t)\right. \\
& \left.\left.+{ }^{2} S^{1}(x, z, t)\right)+O\left(\epsilon_{i}\right)^{2}\right] \\
\frac{\partial \widehat{S}(x, z, t)}{\partial \widehat{x}}= & \frac{S_{0}}{L_{s}}\left[\frac{\partial^{1} S^{0}(x, t)}{\partial x}+\frac{\partial^{0} S^{0}}{\partial x}\right. \\
& +\epsilon_{i}\left(\frac{\partial^{0} S^{1}(x, z)}{\partial x}+\frac{\partial^{1} S^{1}(x, z, t)}{\partial x}\right. \\
& \left.+\frac{\partial^{2} S^{1}(x, z, t)}{\partial x}+O\left(\epsilon_{i}\right)^{2}\right]
\end{aligned}
$$

where: $\widehat{S}$ is the dimensional salinity and $S_{0}$ is the salinity scale $\left(S_{0}=33 \mathrm{ppt}\right) .{ }^{0} S^{0}(x)$ represents the linear mean salinity gradient, and ${ }^{1} S^{0}(x, t)$ is the tidal advection thereof. Stratification is weak and appears only at $O\left(\epsilon_{i}\right)$. Only the tidal and $O\left(\epsilon_{i}\right)$ steady values will be calculated here; ${ }^{0} S^{1}(x, z)$ will be referred to as the salinity defect. Assumption of a spatially uniform horizontal salinity gradient requires that $S_{0}\left({ }^{0} S^{0}(x)\right)$ and $S_{0}\left(\partial^{0} S^{0} / \partial x\right)$ be given by $S_{0}\left(1-\widehat{x} / L_{s}\right)$ and $-S_{0} / L_{s}$, respectively.

The advection of the uniform horizontal density field is calculated using a vertically integrated $O(1)$ salinity equation. The vertically integrated form is obtained by starting from

$$
\frac{\partial \widehat{S}}{\partial \widehat{t}} \cong-\widehat{U}_{t} \frac{\partial \widehat{S}}{\partial \widehat{x}}
$$

in dimensional variables. Vertical advection and turbulent exchange are neglected here because ${ }^{0} S^{0}$ and ${ }^{1} S^{0}$ are assumed independent of $z$. Vertical integration of the nondi- 
mensional form of (19) yields an $O(1)$ integrated tidal salt balance:

$$
\frac{\partial^{1} S^{0}}{\partial t}=+\frac{\mathcal{U}_{b}}{\omega L_{s}} \int_{-1}^{0}{ }^{1} U_{t}^{0} d z
$$

The time integral of (20) with the linear, time-averaged gradient added is

$$
\begin{aligned}
{ }^{1} S^{0}+{ }^{0} S^{0}= & \operatorname{Re}\left[\frac{\mathcal{U}_{b}}{\omega L_{s}} M^{\prime}(x)\right. \\
& \left.\times\left(1+\left.\frac{i}{2 m^{2}} P^{\prime}\right|_{-1}\right) e^{i t}+1-x\right]
\end{aligned}
$$

The assumption of a uniform horizontal density gradient advected by the tides has the interesting result that it produces, because of the along-channel decay in the tidal currents, a small tidal variation in the horizontal density gradient. Because damping length of the tidal wave $(1 / \operatorname{Im}(q))$ is much longer than $L_{s}$, this is not an $O(1)$ phenomenon, but it does suggest a reason for the observed tidal cycle variations in horizontal density gradient in Figures $4 a-4 c$ of part 1 . Water and salt parcels near the entrance are more strongly advected during the tidal cycle than water and salt parcels further upstream. The result is a compression of the salinity contours on flood and a dilation on ebb. As discussed in part 1, the tidal flats near the entrance are responsible for the observed sharp decrease in tidal transport in the upstream direction. The uniform channel, barotropic tidal model used here does not include these tidal flats and underestimates the along-channel transport divergence in the lower part of the Columbia River Estuary; (21) similarly underestimates the tidal cycle variation in horizontal density forcing.

The dimensional equations for the baroclinic mean circulation are

$$
\begin{aligned}
& 0=-g \frac{\partial \widehat{h}_{i}}{\partial \widehat{x}}+g \alpha \frac{\partial \overline{\widehat{S}}}{\partial \widehat{x}} \widehat{z}+\frac{\partial}{\partial z}\left(K_{m} \frac{\partial \widehat{U}_{i}}{\partial \widehat{z}}\right) \\
& \left(\epsilon_{i} \widehat{U}_{i}+\epsilon_{b} \widehat{U}_{\ell}\right) \frac{\partial \overline{\widehat{S}}}{\partial \widehat{x}}+\overline{\widehat{U}_{t} \frac{\partial \widehat{S}}{\partial \widehat{x}}}=\epsilon_{i} \frac{\partial}{\partial z}\left(K_{\rho} \frac{\partial \widehat{\widehat{S}}}{\partial \widehat{z}}\right) \\
& \frac{\partial \widehat{U}_{i}}{\partial \widehat{x}}+\frac{\partial \widehat{W}_{i}}{\partial \widehat{z}}=0 \\
& 0=-\epsilon_{b} \widehat{Q}_{R} \frac{\partial \overline{\widehat{S}}}{\partial \widehat{x}}+\int_{-H}^{0} \overline{\frac{\partial \widehat{S}}{\partial \widehat{x}} \widehat{U}_{t}} d \widehat{z}
\end{aligned}
$$

where

$\widehat{U}_{\ell}$ barotropic Lagrangian velocity, equal to $\widehat{\bar{U}_{t}}+\widehat{U_{S}}$;

$\rho$ density, equal to $\rho_{0}(1+\alpha S)$;

$\alpha$ haline contraction coefficient, approximately equal to $7.8 \times 10^{-4}$;

$K_{\rho} \quad$ vertical mass diffusivity.

These represent, respectively, conservation of momentum, alt, and mass, and integral salt conservation. The baroclinically induced surface elevation ${ }^{0} h_{i}^{1}$ in $(22 a)$ arises be- cause of the definition of the baroclinic flow as a zero transport mode. Without this slope, there would be a net inward transport of water in the baroclinic mode (which would have to be compensated in the barotropic mode) because the baroclinic pressure gradient is inward at all depths. The Lagrangian velocity $U_{\ell}$, rather than the mean Eulerian velocity $\widehat{\bar{U}}_{t}$ appears in $(22 b)$ because salt is advected by the Lagrangian flow, not just the Eulerian flow. The purpose of $(22 d)$ is to specify the salinity intrusion length $L_{s}$ by requiring that salt be conserved in an integral sense. Essentially, $(22 d)$ states that the sum of inward salt transports is balanced against outward transport by the total mean flow. The river flow $Q_{R}$ appears in $(22 d)$ because the vertical integral of the Lagrangian current $\widehat{U}_{\ell}$ is $Q_{R}$ (equation $(10 d)$ ); that is, $Q_{R}$ is the sum of the Stokes drift and the integral of the Eulerian mean flow. The baroclinic velocity $\epsilon_{i} \widehat{U}_{i}$ does not appear in $(22 d)$ because the baroclinic flow is an internal mode which integrates to zero.

The $O\left(\epsilon_{i}\right)$ equations will be solved under the assumptions that the vertical eddy diffusivity $K_{m}$ is constant in the vertical and equal to the mass diffusivity $K_{\rho}$; that is, the turbulent Prandtl number is unity. In nondimensional form, $(22 a)$ to $(22 d)$ are

$$
\begin{gathered}
\frac{\partial^{2}{ }^{0} U_{i}^{1}}{\partial z^{2}}=+\frac{g \zeta_{i} H^{2}}{K_{m} L_{s} \mathcal{U}_{i}} \frac{\partial^{0} h_{i}^{1}}{\partial x}+\frac{H^{3}}{K_{m} \mathcal{U}_{i}} \frac{g \alpha S_{o}}{L_{s}} z \\
\frac{\partial^{2}{ }^{0} S^{1}}{\partial z^{2}}=-\frac{\epsilon_{b}}{\epsilon_{i}} \frac{H^{2} \mathcal{U}_{b}}{K_{\rho} L_{s}}{ }^{0} U_{l}^{1}-\frac{H^{2} \mathcal{U}_{i}}{K_{\rho} L_{s}}{ }^{0} U_{i}^{1} \\
+\frac{1}{\epsilon_{i}} \frac{\mathcal{U}_{b}}{\omega L_{s}} \frac{\partial^{1} S^{0}}{\partial x}{ }^{0} U_{t}^{1} \\
\\
\frac{{ }^{0} U_{i}^{1}}{\partial x}+\frac{\partial^{0} W_{i}^{1}}{\partial z}=0 \\
0=\epsilon_{b} \frac{H^{2} \mathcal{U}_{b}}{K_{\rho} L_{s}} Q_{R}-\frac{\mathcal{U}_{b}}{\omega L_{s}} \int_{-1}^{0} \frac{\frac{\partial^{1} S^{0}}{\partial x}{ }^{1} U_{t}^{0} d z}{{ }^{0}} d z
\end{gathered}
$$

where

$$
\begin{aligned}
& \mathcal{U}_{i}=\epsilon_{i}\left(\frac{\Delta \rho_{h}}{\rho_{0}} g H\right)^{1 / 2}=0.25-0.5 \mathrm{~m} \mathrm{~s}^{-1} \\
& \zeta_{i}=0.1-0.2 \mathrm{~m}=O\left(\epsilon_{i} \zeta\right)
\end{aligned}
$$

The $O(1)$ baroclinic mode is absent; thus the baroclinic velocity scale for the mean flow $\mathcal{U}_{i}$ is $\epsilon_{i}$ times that specified by (16). The baroclinic velocity field and surface slope can be found by integrating (23a) and (23c), using zero stress at the surface, a slip velocity at the bed, and the constraint that the baroclinic flow is a zero transport mode. The result is

$$
\begin{aligned}
&{ }^{0} U_{i}^{1}= r_{1}\left(\frac{z^{3}}{6}+1\right) \\
&+\frac{3}{2}\left(\frac{r_{1}}{8}+{ }^{0} V_{s i}^{1}\right)\left(z^{2}-1\right)+{ }^{0} V_{s i}^{1} \\
&{ }^{0} W_{i}^{1}=0 \\
& \frac{{ }^{0} h_{i}^{1}}{\partial x}=\frac{3\left(r_{1} / 8+{ }^{0} V_{s i}^{1}\right)}{r_{2}}
\end{aligned}
$$


where

$$
r_{1}=\frac{H^{3} g \alpha S_{0}}{K_{m} \mathcal{U}_{i} L_{s}} \quad r_{2}=\frac{g \zeta_{i} H^{2}}{K_{m} \mathcal{U}_{i} L_{s}}
$$

Thus for $r_{1}>>{ }^{0} V_{s i}^{1}$, the baroclinic velocity is proportional to the cube of the depth, the horizontal density gradient, and the inverse of the eddy diffusivity. ${ }^{0} U_{i}^{1}$ depends weakly on $\mathrm{x}$, because of the $x$ dependence of $\partial S / \partial x$ (equation (21)) and $L_{s}$ in $r_{1}$. If $r_{1}$ is taken constant, then continuity requires that the mean baroclinic vertical velocity ${ }^{0} W_{i}^{1}$ vanish everywhere, as it vanishes at the bed.

Integration of $(23 b)$ to determine the salinity defect as a function of depth requires calculation of the nonlinear, tidal advection term

$$
\begin{aligned}
& \overline{{ }^{1} U_{t}^{0}} \frac{\partial^{1} S^{0}}{\partial x}= \\
& \frac{\mathcal{U}_{b}}{\omega L_{s}} \operatorname{Re}\left[\frac{i}{2} M^{\prime \prime *} M^{\prime} P\left(1-\left.\frac{i}{2 m^{2}} P^{\prime *}\right|_{-1}\right)\right]
\end{aligned}
$$

A modified form of the tidal advection will be introduced later, to account for the enhanced advection associated with the presence of tidal flats near the entrance. The requisite boundary conditions are zero salinity defect and zero salt flux at the bed. The salinity defect is then the salinity deviation from the surface salinity specified by the $O(1)$ tidal salinity equation (21). Imposition of integral salinity conservation (equation (24)) allows the additional constraint of zero salt flux at the bed also to be met. Integration of the salt conservation equation $(23 b)$ gives the salinity defect:

$$
\begin{aligned}
{ }^{0} S^{1}= & -\frac{\epsilon_{b}}{\epsilon_{i}} \frac{H^{2} \mathcal{U}_{b}}{K_{\rho} L_{s}} \int_{0}^{z} \int_{0}^{z}{ }^{0} U_{\ell}^{1} d z d z \\
& -\frac{H^{2} U_{i}}{K_{\rho} L_{s}} \int_{0}^{z} \int_{0}^{z}{ }^{0} U_{i}^{1} d z d z \\
& +\frac{1}{\epsilon_{i}} \frac{\mathcal{U}_{b}}{\omega L_{s}} \int_{0}^{z} \int_{0}^{z} \frac{\frac{\partial{ }^{1} S^{0}}{\partial x} U_{t}^{0}}{\partial x} d z
\end{aligned}
$$

The three integrals contributing to the salinity defect are given by

$$
\begin{aligned}
& -\frac{H^{2} \mathcal{U}_{i}}{K_{\rho} L_{s}} \int_{0}^{z} \int_{0}^{z}{ }^{0} U_{i}^{1} d z d z= \\
& -\frac{H^{2} \mathcal{U}_{i}}{K_{\rho} L_{s}}\left[\frac{3}{2}\left(\frac{r_{1}}{8}+{ }^{0} V_{s i}^{1}\right)\left(\frac{z^{4}}{12}-\frac{z^{2}}{2}\right)\right. \\
& \left.+\frac{r_{1}}{6}\left(\frac{z^{5}}{20}+\frac{z^{2}}{2}\right)+{ }^{0} V_{s i}^{1} \frac{z^{2}}{2}\right]
\end{aligned}
$$

$$
\begin{aligned}
& -\frac{\epsilon_{b}}{\epsilon_{i}} \frac{H^{2} U_{b}}{K_{\rho} L_{s}} \int_{0}^{z} \int_{0}^{z}{ }^{0} U_{\ell}^{1} d z d z= \\
& -\frac{\epsilon_{b}}{\epsilon_{i}} \frac{H^{2} U_{b}}{K_{\rho} L_{s}} R e\left\{2 m^{2} \frac{\partial^{0} h_{t}^{1}}{\partial x} \frac{z^{4}}{4}+C_{3} \frac{z^{2}}{2}\right. \\
& +m^{2} M^{\prime \prime *} M^{\prime}\left[\frac{-5 i}{8 m^{2}} z^{2}\right. \\
& +\left(c_{2}-1+\left.\frac{i}{2 m^{2}} P^{\prime *}\right|_{1}\right) \frac{z^{3}}{6}+\frac{z^{4}}{24} \\
& -\frac{19}{16 m^{4}}\left(P-\left.P\right|_{0}\right)-\frac{3}{16 m^{4}}\left(P^{*}-\left.P^{*}\right|_{0}\right) \\
& \left.\left.+\frac{3}{16 m^{4}}\left(|P|^{2}-\left.|P|\right|_{0} ^{2}\right)-\frac{3 i}{32 m^{6}}\left|P^{\prime}\right|^{2}\right]\right\} \\
& \frac{1}{\epsilon_{i}} \frac{\mathcal{U}_{b}}{\omega L_{s}} \int_{0}^{z} \int_{0}^{z} \overline{\frac{{ }^{1} S^{2}}{\partial x}{ }^{0} U_{t}^{1}} d z d z= \\
& -\frac{1}{\epsilon_{i}}\left(\frac{\mathcal{U}_{b}}{\omega L_{s}}\right)^{2} R_{e}\left[\frac{i}{2} M^{\prime \prime *} M^{\prime}\right. \\
& \left.\times\left(1-\left.\frac{i}{2 m^{2}} P^{\prime *}\right|_{-1}\right)\left(\frac{z^{2}}{2}-\left.\frac{i}{2 m^{2}} P\right|_{-1} ^{0}\right)\right]
\end{aligned}
$$

The salinity defect is the sum of terms arising from products of the mean horizontal salinity gradient with the mean barotropic and mean baroclinic velocities, and of the tidal salinity gradient with the tidal velocity. The barotropic effect may be further broken down into parts driven by the Stokes drift velocity and the mean Eulerian velocity. The first integration shows that the salinity gradient at any depth due to any of these effects is controlled by the sign of the transport between the surface and that depth. The baroclinic flow, since it is a zero transport mode which is outward at the surface, will yield a stable stratification except at the bed, where the salinity defect gradient goes to zero as the transport integrates to zero. The Eulerian mean flow transport also yields a stable salinity gradient, so long as there is a nonzero (outward) river flow. The tidal advective salt transport term, however, gives a tendency to vertical instability. The vertically integrated salt balance of $(24)$ requires that the vertical salt flux (and thus the total salinity defect gradient) vanish at the surface and the bed. This requirement determines the mean salinity intrusion length $L_{s}$ (and thus the value of the mean salinity gradient) so that there is no net loss or gain of salt. The two barotropic contributions to the salinity defect gradient must then cancel one another at the bed.

\section{Modifications and Additions to the Analytical Solutions}

There are four areas in which the barotropic and baroclinic solutions presented above require modifications or 
additions, so that predictions can be made for the Columbia River Estuary. First, the baroclinic solution is only applicable at those times and places where a salinity gradient is present. That is, the computer code that implements the analytical solution must distinguish between oceanic, estuarine, and fluvial water masses. Second, the analytical solutions do not specify the value of coefficients of vertical exchange of momentum and mass. This is particularly critical in that the stratification calculated from the baroclinic solution reduces the vertical mixing below that expected in a neutrally stratified flow. Third, the slip velocity in each part of the analytical solution must be estimated. Finally, the analytical solutions, while correct for a channel of uniform cross section, do not reproduce the dilation and contraction of the density field during the tidal cycle that is observed in the prototype system. This variability in the horizontal density gradient is caused by the along-channel variability in transport induced by the presence of large tidal flats in the lower estuary.

Determination of the proper eddy and mass diffusivities requires iteration between the barotropic and baroclinic models. The vertically integrated barotropic transport model of (10) of part 1 can be used to provide an initial estimate of the neutral eddy diffusivity $K_{n}$ (assumed constant through the tidal cycle) by expressing the boundary shear stress $u_{*}^{2}$ in terms of the tidal cycle maximum velocity ${ }^{1} U_{m}^{0}$ :

$$
\begin{gathered}
{ }^{1} U_{m}^{0}=\left.\left.\right|^{1} Q^{0}\right|_{x=0} \mid \\
\widehat{u}_{*}^{2}=c_{d} \mathcal{U}_{b}^{2}\left({ }^{1} U_{m}^{0}\right)^{2} \\
K_{n}=\frac{k \widehat{u}_{*} H}{\Gamma}
\end{gathered}
$$

where $c_{d}$ is the drag coefficient and $\Gamma=6.24$. This (constant) value of $K_{n}$ was used by Rattray and Mitsuda [1974] for the outer part of the flow. It was matched to a linear profile close to the bed at a matching height $z_{m}=-0.8$ and is based on pipe flow measurements of Hinze [1959]. In the present analysis, the eddy diffusivity is assumed constant with depth, and the no-slip condition is relaxed instead. This initial estimate of the eddy diffusivity $K_{m}$ (and of the mass diffusivity $K_{\rho}$, assuming a turbulent Prandtl number of unity) is refined by iteration between the baroclinic and barotropic parts of the model, using a stratification correction based on a gradient Richardson number $R i_{g}$ formed from the stratification in the mean flow and the shear in the total flow. The stratification correction $S_{c r}$ used is that defined by Long [1981]:

$$
\begin{gathered}
K_{m}=K_{n} S_{c r} \\
S_{c r}=\left(1-\beta R i_{f}\right)
\end{gathered}
$$

where $R i_{f}$ is the flux Richardson number and is equal to $R i_{g}$ for $K_{\rho} / K_{m}=1$. The value of $\beta(=4.7)$ used herein is that suggested by Businger et al. [1971]. Wieringa [1980] has argued for $\beta=6.9$, but the difference is of minimal importance in this context.

Because $S_{c r}$ and $R i_{g}$ are functions of depth, it is necessary to choose the correction at a particular depth $\left(z_{s}\right)$ to use with the vertically uniform eddy diffusivity in the analytical model. We assume that the most appropriate depth lies between the maximum in Long's neutral eddy diffusivity profile at about $z=-0.84$ and about middepth $(z=-0.5)$. Below $z=-0.84$, turbulent eddy size is directly dependent on distance from the boundary, and as the bed is approached, strong shear and strong vertical mixing will be present under all conditions. At the free surface, the flow will always be inviscid so long as the free surface is unstressed, and profile data (e.g., Figure 6 of part 1) suggest that much of the outer half of the flow is inviscid even when mixing is strong throughout the remainder of the water column. A depth of $z_{s}=-0.25$ was therefore adopted for the model runs presented here.

The slip velocities $V_{s}$ in each analytical solution is the constant that must be added to a parabolic velocity profile so that the parabolic profile and a log profile (both with the same friction velocity as the analytical solution) are equal at the matching depth $z_{m}$. In each case, the appropriate friction velocity has been defined from a vertically integrated force balance. This procedure corresponds to the assumption that the time-dependent flow is governed, at peak flood or ebb, by a balance of pressure gradient versus stress divergence. Thus for the $O(1)$ tidal flow,

$$
\begin{aligned}
{ }^{0} V_{s t}^{1}=\frac{u_{*}}{k} & {\left[\Gamma\left(\frac{\left(z_{m}+1\right)^{2}}{2}-z_{m}-1\right)\right.} \\
& \left.-\ln \left(\frac{z_{m}+1}{z_{0}}\right)\right]
\end{aligned}
$$

where ln is the natural logarithm and $k$ is von Kármán's constant, equal to $\mathbf{0 . 4}$. To evaluate the correct matching depth, numerical experiments have been made using matching depths between $z=-0.9$ and $z=-0.8$. Differences in flow profiles and slip velocities were minor, and $z_{m}=-0.85$ was adopted for further runs. The bed roughness has been specified as $z_{0}=0.00065 H$, a typical value for large tidal rivers with fully developed bed forms.

Correct reproduction of the along-channel variation in tidal transport, as modified by the presence of tidal flats, is necessary to achieve the observed tidal dilation and contraction of the density field during the tidal cycle (Figure $4 a$ and $4 b$ of part 1 ). Correct specification of this dilation and contraction is in turn necessary to calculate the tidal correlation contribution to the salinity defect. It can readily be shown [Perroud, 1958] that an exponentially decreasing width of the form $b=b_{0} e^{-\gamma_{1} x}$ contributes a $e^{-\gamma_{1} x / 2}$ term to the tidal transport. The modified forms of the $O(1)$ salinity and salinity gradient are

$$
\begin{aligned}
& { }^{1} S^{0}+{ }^{0} S^{0}= \\
& \operatorname{Re}\left[\frac{\mathcal{U}_{b}}{\omega L_{s}} M^{\prime} \Psi\left(1+\left.\frac{i}{2 m^{2}} P^{\prime}\right|_{-1}\right) e^{i t}+1-x\right]
\end{aligned}
$$




$$
\begin{aligned}
\frac{\partial^{1} S^{0}}{\partial x}+\frac{\partial^{0} S^{0}}{\partial x} & =\operatorname{Re}\left[\frac{\mathcal{U}_{b}}{\omega L_{s}}\left(M^{\prime \prime} \Psi+M^{\prime} \Psi^{\prime}\right)\right. \\
& \left.\left(1+\left.\frac{i}{2 m^{2}} P^{\prime}\right|_{-1}\right) e^{i t}-1\right]
\end{aligned}
$$

where

$$
\Psi=\left(1+\gamma_{2} e^{-\gamma_{1} / 2 x}\right)
$$

$\gamma_{1} e$-folding factor for the channel width, $\sim \frac{1}{40} \mathrm{~km}$;

$\gamma_{2}=\left(b_{t}-b_{s}\right) / b_{s} \approx 0.4$;

$b_{t}$ total width;

$b_{s}$ stream width.

The values of $\gamma_{1}$ and $\gamma_{2}$ are chosen on the basis of actual along-channel variations in tidal flat width for the Columbia River Estuary. Using this form of the salinity gradient, a revised tidal correlation salinity defect contribution can be calculated.

The above modifications and additions to the analytical solution allow realistic simulations of the velocity and density field in the Columbia River Estuary.

\section{Results: Properties of Weakly Stratified Estuarine Flows}

\section{Tidal Circulation}

Basic properties of the weakly stratified estuarine flows were investigated using a nondimensional river flow of $Q_{R}=0.1$ to 0.15 (as in part 1) and tidal amplitudes $H_{0}=1.0$ to $2.5 \mathrm{~m}$. Typical peak flood flows are shown in Figures $1 a$, and $1 b$ and peak ebb flows are shown in Figures $2 a$, and $2 b$ for $Q_{R}=0.15$ and $H_{0}=1.7 \mathrm{~m}$. The position of $x=0$ is set to the entrance bar at RM(river mile)-1.5 (km-2.4).

The predicted flood and ebb velocity fields (Figures $1 b$ and $2 b$ ) can be interpreted in terms of the harmonic decomposition that is the basis of the model calculations. The tidal-frequency flow is perfectly reversing at every point. The entire effect of the ebb-flood asymmetry in
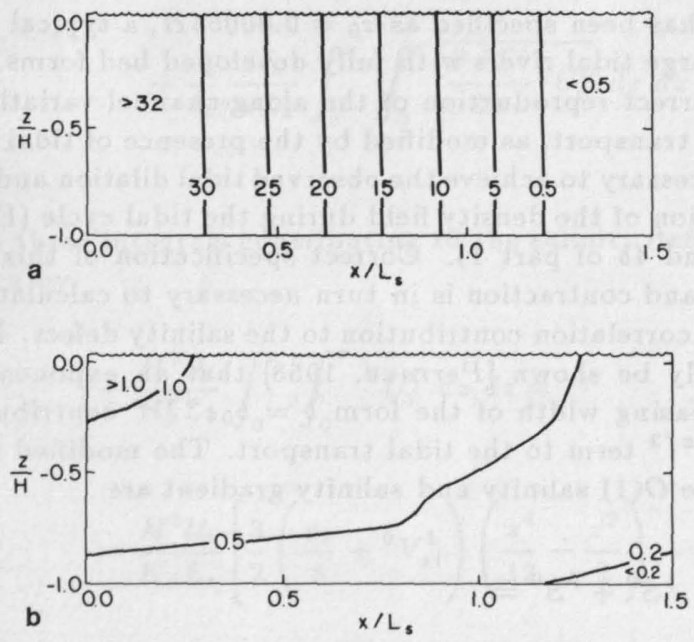

Fig. 1. Weakly stratified flow model predictions of $(a)$ salinity in parts per thousand and $(b)$ along-channel velocity in meters per second for peak flood. Top to bottom stratification is 0.1 to $0.2 \mathrm{ppt}$ in Figure $1 a$.
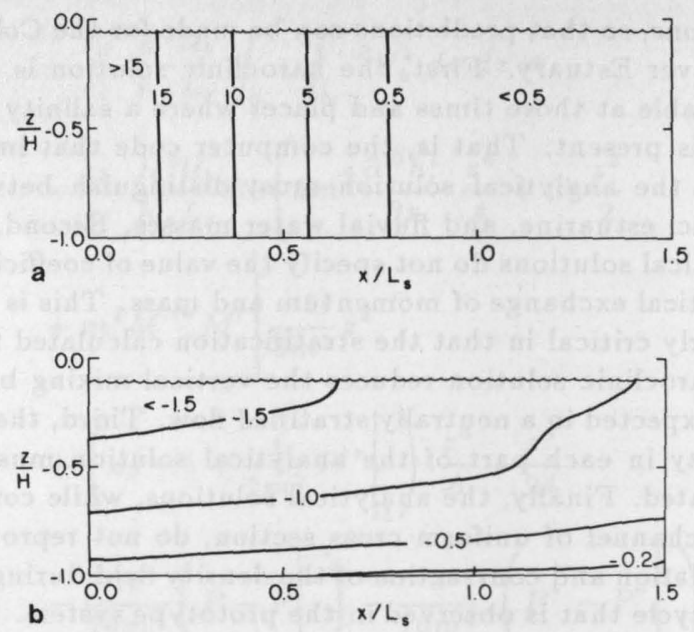

Fig. 2. Weakly stratified flow model predictions of salinity $(a)$ in parts per thousand and $(b)$ along-channel velocity in meters per second for peak ebb. The shear is larger than on the flood, because the mean shear and the tidal shear have the same sign on ebb, whereas they are of opposite sign on flood. Note the floodebb variation of the horizontal density gradient, as discussed in the text.

the model then, stems from the mean flow. Velocities and shears are greatest on ebb, because the tidal shear and velocity are augmented by the mean flow. In contrast, the mean flow (outward, except very close to the bed) reduces on flood both the total velocity and total shear. The predicted density field in Figures $1 a$ and $2 a$ reproduces very well the observed advection and the dilation and contraction of the density field during the tidal cycle (Figures $4 a$ and $4 b$ of part 1 ). The model underestimates the stratification, which is several parts per thousand in the prototype but only 0.1 to $0.2 \mathrm{ppt}$ in the model. This can be explained in terms of the errors introduced by the assumption in the model of an eddy diffusivity $K_{m}$, constant in the vertical. The calculated gradient Richardson number $R i_{g}$ is subcritical throughout the entire flow up to about $z=-0.1$, and the turbulence is strongly inhibited only in the outer $10-20 \%$ of the flow, as indicated by the calculated stratification correction $S_{c r}=1-\beta R i_{g}$ (Figure 3 ). It is believed that the flow and density field near the bed are accurate, because the tidal amplitude at which the spring-neap transition occurs is correctly predicted, as discussed below. In reality, however, even moderate stratification suffices to render the outer part of the flow inviscid (e.g., in Figure 6 of part 1), allowing enhanced shear and stratification to develop. This situation is portrayed schematically by the hypothetical "correct" $R i_{g}$ and $S_{c r}$ curves in Figure 3, showing complete inhibition of turbulence in the outer $40 \%$ of the flow. The predicted shear and stratification near the times of slack water are probably also less than those observed throughout the water column, because of the weak mixing at these times. Abraham [1988] has concluded, however, that even sophisticated turbulence models have difficulty calculating properties at slack water, because the weakening of the tidal flow allows Kelvin-Helmholtz instabilities that are not represented in most turbulence models to be temporarily important. Thus even a large increase in computational effort over the present model may not greatly improve the calculated density field. 


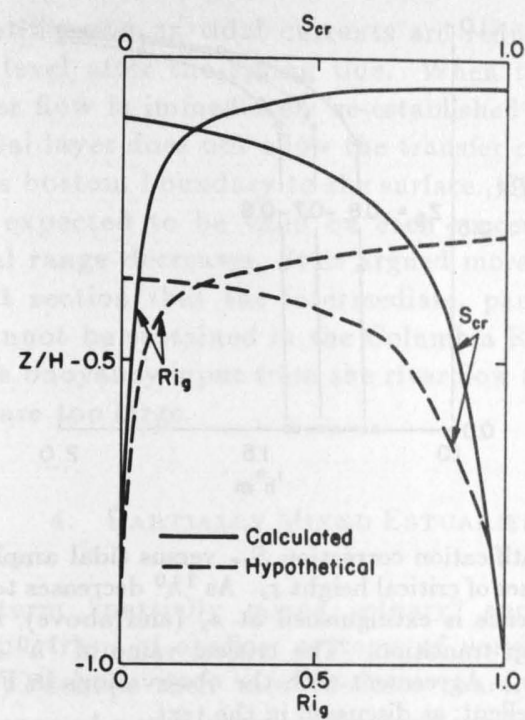

Fig. 3. Model predictions and hypothetical correct values of $R i_{g}$ and $S_{c r}$ for peak flood at $x=0$. The model predicts that only the outer $10 \%$ of the flow should be inviscid, which is an underestimate. The error arises from the assumption of the constant (in the vertical) eddy diffusivity (see text).

\section{Generation of the Residual Circulation}

The residual density and flow fields predicted by the model are shown in Figures $4 a$ and $4 b$. The model does well in representing the mean, along-channel salinity variations but, as for the flood and ebb flows, less well in reproducing the stratification. The decrease in mean horizontal salinity gradient at either end of the estuary is, however, well reproduced. This weakening is caused by the presence of horizontally uniform water masses (either oceanic or fluvial) during part of the tidal cycle. In the prototype there is undoubtedly also a decrease in the mean salinity gradient at the two ends of the system caused by the continuous nature of the instantaneous horizontal salinity gradient at either end of the system; no attempt was made to reproduce this effect in the model. The rms error in predicting the salinity at the 12 available current meter locations is $2.1 \mathrm{ppt}$, relative to a salinity difference of 33 ppt between fresh and salt water. Almost all of this error occurs at the three meters at $x \cong 0.5$. The predicted salinity gradient is too small seaward of this station and too large landward of it. It is not known whether this error is associated with an incorrect representation of tidal flat effects on water transport, topographic irregularities in the channel, or salt input from peripheral channels.

The model velocity field (Figure $4 b$ ) correctly predicts the sign of the velocity at all meters, and it correctly predicts that the vertical shear and upstream bottom flow in the seaward half of the channel are much reduced relative to the two-layer case (Figure $11 b$ of part 1 ). The observations at $x=0.12$, which show an outflow stronger than predicted by the model, should be viewed in light of observations reported by Jay and Smith [1989], which show the cross sectional variation in the mean along-channel velocity in the lower estuary. As discussed in part 1 the meters at $x=0.12$ are in a location strongly favoring outflow. Net inflow occurs elsewhere in the section. Velocity observations for the bridge-mounted meters at $x=0.5$ are not plotted because of a systematic error introduced by
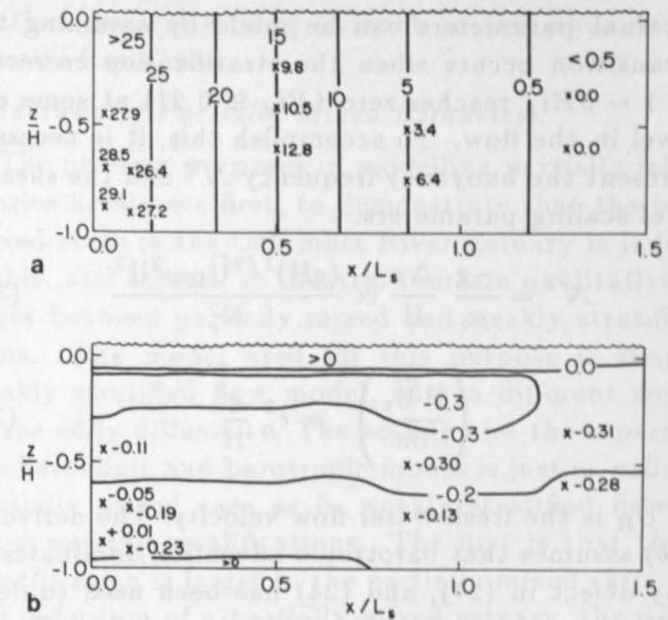

Fig. 4. Model predictions of $(a)$ residual salinity and $(b)$ alongchannel velocity. Observations (50-hour averages) from 12 current meters are included for comparison. Data from the three bridgepier mounted meters at $x=0.51$ are omitted from Figure $4 b$, because of a systematic ebb bias. The model underestimates the stratification as discussed in the text; the rms salinity error is 2.1 ppt. It correctly predicts that the shear should be much less than in the highly stratified case. Upstream bottom flow is therefore much reduced. Net upstream flow near the surface results from the correlation of velocity and tidal phase; the surface level is above mean lower low water (MLLW) mainly on the flood. The two meters near $x=0.07$ were in a location having a strong ebb flow. Upstream flow occurs elsewhere in the section.

the bridge pilings. The maximum upstream bottom flow in the model is 0.01 to $0.02 \mathrm{~m} \mathrm{~s}^{-1}$, which reaches to about $x=0.75$. Although available current meter measurements (all made at least 1 to $2 \mathrm{~m}$ above the bed) do not show it, it is likely that upstream bottom flow occurs very close to the bed in a large part of the channel. This is demonstrated by the sidescan sonar observations of bed forms by Sherwood et al. [1989]. Bed forms with landward oriented slip faces (indicative of landward movement) were found in the bottom of the South Channel during the low-flow season as far upstream as about $x=0.6$. Thus net upstream bed load sediment transport and (since overtide effects are minimal in the lower estuary) presumably net upstream bottom flow occurred at depth during the low-flow season but were absent some distance above the bottom. These observations do not definitely establish whether this movement took place on spring or neap tide or how spatially continuous it was, but at least some movement on spring tides is likely. This supports the correctness of the model predictions in Figure $4 b$.

\section{Stability of Weakly Stratified Estuarine Flow and Spring- Neap Transitions}

Perhaps the most severe test of the weakly stratified flow model is prediction of the tidal amplitude of the spring-neap transition. As was the case in the two-layer model, it is the structure of the flow on flood which is the critical factor. That is, because of the stronger flows and greater shears, $R i_{g}$ will remain subcritical on ebb even when the tidal forcing is small and a weakly stratified flow cannot be maintained on the flood. A scale argument to determine the dependence of the spring-neap transition 
on external parameters can be made by assuming that this transition occurs when the stratification correction $S_{c r}=1-\beta R i_{g}$ reaches zero $\left(R i_{g} \cong 0.21\right)$ at some critical level in the flow. To accomplish this, it is necessary to represent the buoyancy frequency $\mathcal{N}^{2}$ and the shear in terms of scaling parameters:

$$
\begin{gathered}
\mathcal{N}^{2}=\frac{-\mathrm{g}}{\rho_{\mathrm{o}}} \frac{\Delta \rho}{\mathrm{H}} \propto \frac{(\mathrm{gH})^{1 / 2} \widehat{\mathrm{U}}_{\mathrm{R}} \omega^{2} \mathrm{H}^{2}}{\mathrm{~K}_{\rho}^{2}} \\
\left(\frac{\partial \widehat{\mathrm{U}}_{\mathrm{t}}}{\partial \mathrm{z}}\right)^{2} \propto \epsilon_{\mathrm{b}}^{2} \frac{\mathrm{g}}{\mathrm{H}}
\end{gathered}
$$

where $\widehat{U}_{R}$ is the fresh water flow velocity. The derivation of $(34 b)$ assumes that barotropic advection dominates the salinity defect in (27), and (24) has been used to define $L_{s}$. The assumption that $K_{\rho}=K_{m}$ (a turbulent Prandtl number of unity), and equations (29b) and (29c) yield an expression for $R i_{g}$ :

$$
R i_{g} \propto \frac{\widehat{U}_{R}}{\mathcal{U}_{b}} \epsilon_{b}^{-1} m^{4}=\frac{\Gamma^{2}}{4 k^{2} c_{d}\left({ }^{1} U_{m}^{0}\right)^{2}} \frac{\widehat{U}_{R}}{\mathcal{U}_{b}} \epsilon_{b}^{-3} \frac{\omega^{2} H}{g}
$$

$R i_{g}$ varies linearly with the nondimensional river flow $\widehat{U}_{R} / \mathcal{U}_{b}$ and inversely with the cube of nondimensional tidal amplitude $\epsilon_{b}$. In deriving this expression it has been assumed that $K_{m} \cong K_{n}$ up to the point at which the spring-neap transition occurs. This assumption is justified in the next paragraph.

The behavior of the model as the tidal range is decreased has been examined to obtain a prediction of the critical tidal amplitude that may be compared to observations. This analysis requires the iterative determination of $S_{c r}$ at a specified critical level. The $18 \%$ of the flow below the maximum in the neutral eddy diffusivity profile [Long, 1981] can be excluded from consideration in this regard, because turbulent properties are strongly dependent on the presence of the bed, and this part of the flow remains turbulent even when the tidal range is severely reduced. Similarly, the outer half of the flow may become inviscid without strongly affecting the properties of the flow as a whole, as is demonstrated by the profile data (e.g., Figures $6 a$ and $6 b$ of part 1). We are therefore concerned with the flow between roughly $18 \%$ and $50 \%$ of depth. Three different iteration depths and a variety of tidal ranges at each depth were used. The depths of these were $z_{s}=-0.6,-0.7$, and -0.8 . The results are quite striking (Figure 5). Regardless of the value of $z_{s}, S_{c r}$ remains above about 0.7 so long as as the tidal range is above a critical level. At a certain critical tidal amplitude which depends on $z_{s}$, a very small further decrease in tidal amplitude ( $<0.02 \mathrm{~m}$ in every case) suffices to completely extinguish turbulent mixing at the iteration depth. These tests specify the critical tidal amplitude of the spring-neap transition as being between 1.22 and $1.42 \mathrm{~m}$. Because the spring-neap transition takes several days during a period when the tidal range is changing rapidly, it is not possible to determine exactly the critical tidal amplitude from observations. Figure 1 of part 1 , however, suggests a value of between 1.1 and $1.4 \mathrm{~m}$; this result constitutes a strong confirmation of the theory.

A reviewer questioned whether the transition criterion derived above is identical to the $\widehat{U}_{t}^{3} / H=P_{\text {crit }}$ criterion of Simpson and Hunter [1974]. Indeed there is a similarity

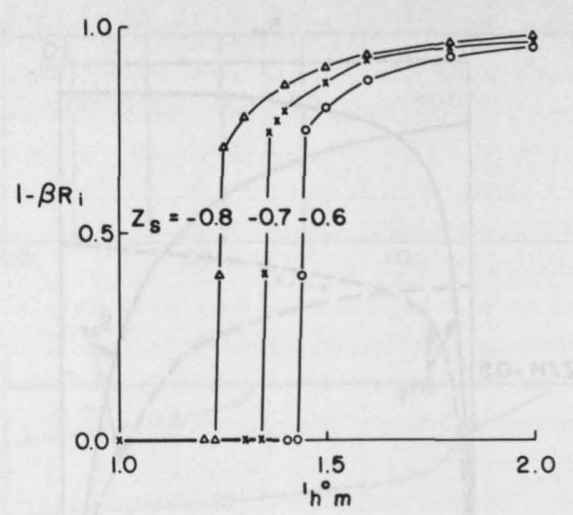

Fig. 5. Stratification correction $S_{c r}$ versus tidal amplitude ${ }^{1} h^{0}$ for three values of critical height $z_{s}$. As ${ }^{1} h^{0}$ decreases to a critical level, turbulence is extinguished at $z_{s}$ (and above), leading to a spring-neap transition. The critical value of ${ }^{1} h^{0}$ is a weak function of $z_{s}$. Agreement with the observations in Figure 1 of part 1 is excellent, as discussed in the text.

in conceptual approach: the $\widehat{U}_{t}^{3} / H$ criterion is based on the turbulent kinetic energy balance, with dissipation at the bed taken equal to be a specified fraction of production over the water column. The remaining production is assumed go into increasing the potential energy of the flow through the buoyancy flux. There are, however, vital differences as well. First, because the Simpson and Hunter approach is effectively based on a bulk Richardson number, its critical value (equivalent to the efficiency term in $\left.P_{\text {crit }}\right)$ may vary somewhat, depending on the nature of the flow. More importantly, $\widehat{U}_{t}^{3} / H$ contains no information concerning the buoyancy flux - this must be known to specify $P_{\text {crit }}$. Determination of the buoyancy flux in an estuary requires knowledge of $\widehat{U}_{R}$ and $L_{s}$, and $L_{s}$ is one of the unknowns that must be calculated by any model of estuarine circulation. Thus the present model is closed in a sense that the $\widehat{U}_{t}^{3} / H=P_{\text {crit }}$ criterion is not, and a prediction of the neap-spring transition using $\widehat{U}_{t}^{3} / H$ is not trivial, because $P_{\text {crit }}$ depends in a nonlinear manner on the tidal range through $L_{s}$.

The discussion up to this point has not considered the nature of the intermediate, partially mixed state which appears during the tidal monthly transitions. Several reasons may be advanced as to why the it lasts only a few days. First, the flushing time of the system is sufficiently short (1 to 3 days, [Neal, 1972]) relative to the tidal month that the density field responds to the daily variations in tidal range, not to some long-term average. Second, each of the two states of the density field leads directly to the other through the transition mechanisms previously discussed. For example, the boundary layer depth scale defined by $\widehat{u}_{*} /(2 \omega)$ is substantially greater than the depth of flow. Therefore, mixing during any single flood after the neap-spring transition should bring about a weakly stratified state during that flood. This situation will pertain more strongly on each successive tide, so long as the tidal range increases. Nor is this conclusion sensitive to the nature of the entrance density boundary condition, so long as the density difference between seawater and fresh water is not made unrealistically large. Rather, it depends only on well-defined conditions inside the estuary. Conversely vertical mixing at mid-depth on flood is extinguished by 
the stratification as tidal currents are reduced below a critical level after the spring tide. When this occurs a two-layer flow is immediately re-established because the interfacial layer does not allow the transfer of turbulence from the bottom boundary to the surface. This condition can be expected to be valid on each successive tide as the tidal range decreases. It is argued more formally in the next section that the intermediate, partially mixed state cannot be sustained in the Columbia River because both the buoyancy input from the river flow and the tidal forcing are too large.

\section{Partially Mixed Estuaries}

\section{Definition}

The term "partially mixed estuary" encompasses a broad spectrum of shallow systems of varying size and geometry.Perhaps their most definite characteristics are the presence of a well-developed mean baroclinic flow and relatively weak vertical mixing. Near-bottom inward and near-surface outward transports are typically much larger than the net Eulerian transport [Dyer, 1973]. We propose here a more specific definition, one based on vertical exchange processes: a partially mixed estuary is a shallow system wherein the gradient Richardson number $R i_{g}$ is large enough that boundary-induced turbulence is damped in the interior, but wherein stratification is insufficient to support a large, tidal-frequency internal circulation. It is a well-known consequence of turbulent energets that whenever the flux Richardson number $R i_{f}$ exceeds critical value, turbulence cannot be sustained. Morever, tidal-frequency, internal oscillations (and, therefore, igorous interfacial exchange driven by internal shear) must be absent from partially mixed estuaries, as from weakly stratified estuaries. The dynamical reasons such oscillations do not exist in partially mixed estuaries are just as in section 2 - the gravitational restoring force is too weak to sustain such an oscillation.

We suggest that the primary mechanism of vertical exchange on the flood is, in the absence of boundary induced turbulence and strong Kelvin-Helmholtz instabilities, the random interaction and consequent breaking of internal waves. These interactions are not absent in the weakly stratified and highly stratified regimes discussed in sections 2 and 3 and in part 1 , but in both of these cases, the vertical exchange of salt and fresh water is caused primarily by other, more energetic processes. Random internal wave interactions become important only when their presence is not masked by stronger processes. This hypothesis concerning the dominant vertical mixing process cannot at present be proven, because detailed turbulent flux measurements have not yet been carried out in a partially mixed estuary. Thus while there is little question that internal wave breaking does occur in partially mixed estuaries [e.g., Sarabun et al., 1984], a complete dynamical picture is not available, and it is difficult to exclude other possible mechanisms. In the absence of definite evidence that requires the consideration of lateral mixing or other processes, it is desirable and correct to avoid any assumption that renders the problem fully three-dimensional. Moreover, for the approximate calculations carried out here, the major requirement is that the vertical eddy diffusivity be appropriately constrained so that vertical mixing is much weaker than in weakly stratified estuaries.

\section{Modelling of Partially Mixed Estuaries}

The primary purposes of modelling partially mixed estuaries herein are first, to demonstrate that the partially mixed state in the Columbia River Estuary is indeed unstable, and second, to illustrate certain qualitative differences between partially mixed and weakly stratified systems. The model used for this purpose is simply the weakly stratified flow model, with a different treatment of the eddy diffusivity. The scaling and the separation of the baroclinic and barotropic modes is just as valid in the partially mixed case as in weakly stratified flows, with three possible qualifications. The first is that the mean stratification is larger in the partially mixed case. Within the definition of a partially mixed estuary, the perturbation parameter $\epsilon_{i}=\left(\Delta \rho_{v} / \Delta \rho_{h}\right)^{1 / 2}$ may approach, but not exceed, unity. Thus the model remains at least approximately valid so long as the system remains partially mixed rather than highly stratified. The second problem is the vertical tidal advection of the mean stratification. As the stratification becomes less uniform in the vertical, its tidal advection may become more important. As a rule, however, tidal forcing is weaker in partially mixed estuaries than in either of the classes previously considered, so the vertical advection of the mean salinity will be neglected as before.

The final qualification involves the actual form of the $K_{m}$ and $K_{\rho}$ profiles and the approximate analytical method used here, which assumes a vertically uniform eddy diffusivity. Gargett and Holloway [1984] and Gargett [1984] have argued on the basis of both scaling arguments and data that the effective mass diffusivity in a random internal wave mixing regime is inversely dependent on the stability frequency $\mathcal{N}$, so that $K_{p}=a_{0} \mathcal{N}^{-p}$. The constant $a_{0}$ is environment-dependent, and $p$ may be $1 / 2$ or 1 , depending on the form of the vertical velocity variance in the internal wave field. This relationship implies that the eddy diffusivity should vary over the depth of flow, being larger near the bed and surface, where $\mathcal{N}$ is small, and small in the interior. In reality, $K_{\rho}$ and $K_{m}$ approach their boundary layer values near the bed and are limited by the eddy scale near the free surface. However, the relevant vertical exchange processes are not yet sufficiently well understood to formulate a detailed model of $K_{m}$ and $K_{\rho}$ for the partially mixed state. In the absence of such a model, there is little point in using numerical integration to make detailed predictions for the partially mixed state.

\section{Model Predictions}

The first issue explored with the model is the stability of the partially mixed state in the Columbia River Estuary. To demonstrate that the partially mixed state is unstable in the Columbia River Estuary because the buoyancy input (the river flow) is too large, a precise value of $K_{m}$ and $K_{\rho}$ is not required. Scaling arguments based on (23) and (28a) suggest that if baroclinic advection dominates the salinity defect, the nondimensional vertical density difference is

$$
\frac{\Delta \rho_{v}}{\rho_{o}} \propto \frac{g\left(\Delta \rho_{0} / \rho_{0}\right) H^{9} \omega^{4} U_{f}^{2}}{K_{\rho}^{3} K_{m} \epsilon_{b}^{2}(g H)^{2}}=\frac{\Delta \rho_{0}}{\rho_{o}}\left(\frac{\widehat{U}_{R}}{\mathcal{U}_{b}}\right)^{2} m^{8}
$$


where $\Delta \rho_{0}$ is the density difference between fresh and salt water. Thus $\Delta \rho_{v} / \rho_{0}$ is (for a turbulent Prandtl number of unity) proportional to the square of the nondimensional river flow and the inverse fourth power of $K_{\rho}$ (equation (35)) or, if barotropic advection dominates the salinity defect, the inverse square (equation $(34 a)$ ) of $K_{\rho}$. A minimum estimate of the stratification then requires only an upper limit on $K_{m}=K_{\rho}$. This may be found by estimating $\mathcal{N}^{2} \cong 0.005 \mathrm{~s}^{-2}$, assuming uniform stratification and a top-to-bottom salinity difference of $10 \mathrm{ppt}$. This underestimates $\mathcal{N}$ at mid-depth and so overestimates $K_{\rho}$. Conservative tracer and salt budget methods applied to fjords suggest a $K_{\rho}$ of between about $8 \times 10^{-7}$ and $3 \times 10^{-6} \mathrm{~m}^{2}$ $\mathrm{s}^{-1}$ for this $\mathcal{N}$ [Gargett, 1984]. These values of the mass diffusivity are almost certainly too small for a tidal flow, emphasizing the sensitivity of $a_{0}$ to the specific environment. Pritchard [1967] has used the salt balance in the James River Estuary to estimate $K_{\rho} \cong 0.5$ to $8 \times 10^{-4} \mathrm{~m}^{2}$ $\mathrm{s}^{1}$ as a function of depth in a partially mixed estuary. These estimates are difficult to interpret in that they are based on tidal-cycle averages in a system with substantial tidal-cycle variation. Moreover, the tidal advective contribution to the horizontal salt flux is neglected, introducing an error of unknown magnitude. If the tidal advective salt transport were inward, however, this would reduce the calculated value of $K_{p}$.

To achieve an overestimate of the mixing, $K_{m}$ and $K_{\rho}$ were specified in different runs as $1,4,10$, and $20 \times 10^{-4}$ $\mathrm{m}^{2} \mathrm{~s}^{-1}$. The result in every case except the last was a predicted top-to-bottom stratification much greater than the density difference between fresh water and seawater. Only with $K_{m}=20 \times 10^{-4} \mathrm{~m}^{2} \mathrm{~s}^{-1}$ was the predicted vertical salinity difference reduced to about $30 \mathrm{ppt}$. The model suggests, then, that as soon as bottom boundary turbulence is incapable of penetrating in to the flow, the stratification will become large enough that a two-layer flow is again possible. Further, (35) shows that given fixed values of $\mathcal{U}_{b}, H$, and $\Delta \rho_{0} / \rho_{0}$, it is the strong river flow relative to the tidal flow that renders the partially mixed state unstable in the Columbia River during the springneap transition. The partially mixed state is also unstable, however, during periods of increasing tidal range, i.e., during the neap-spring transition. This can also be understood in terms of (35). Given fixed values of $\widehat{U}_{R}, H$, and $\Delta \rho_{0} / \rho_{0}, \mathcal{U}_{b}$ is too large and thus $m^{2}=\left(\omega H^{2}\right) /\left(2 K_{m}\right)$ too small to sustain a partially mixed state.

The qualitative properties of the partially mixed regime may be investigated by taking a value of within the range found by Pritchard [1967] and reducing the river flow and tidal forcing relative to the Columbia River Estuary. Figures $6 a$ and $6 b$ show the mean density and velocity fields for a system of the same depth as the Columbia, but with a tidal amplitude of $1 \mathrm{~m}$, a river flow velocity of $10^{-3} \mathrm{~m}$ $\mathrm{s}^{-1}$, and $K_{m}$ equal to $0.0004 \mathrm{~m}^{2} \mathrm{~s}^{-1}$. The result is topto-bottom stratification of about $9 \mathrm{ppt}$ in mid-estuary and upstream bottom flows of up to $0.07 \mathrm{~m} \mathrm{~s}^{-1}$. The salinity intrusion length is also enhanced relative to the weakly stratified case; $L_{s} \cong 50 \mathrm{~km}$, and the upstream limit of salinity intrusion on flood is at $\mathbf{k m - 7 0}$. As before, net upstream flow is found at the surface, where water is present only on flood. There is also an apparent anomaly in the flow near the upstream limits of salinity intrusion. This arises because of the difference in eddy diffusivities between the estuary and the river, where $K_{m}=0.032 \mathrm{~m}^{2}$
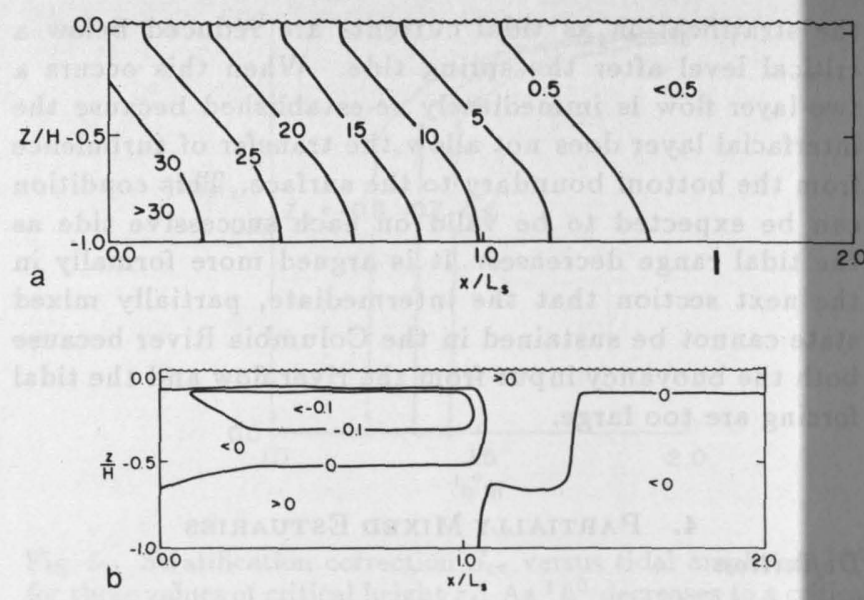

Fig. 6. (a) Predictions of residual salinity and (b) along-channel component of velocity for a hypothetical, partially mixed estuary with tidal forcing similar to the Columbia, but with a river flow smaller by 2 orders of magnitude. Stratification, salinity intrusion length, and net upstream bottom flow are greatly increased relative to Figure 4 . The structure of the velocity contours between $x=1.1$ and $x=1.3$ is caused by a strong flood-ebb difference in $K_{m}$ and therefore in vertical momentum transfer.

$\mathbf{s}^{-1}$ (from $(29 a)$ to $(29 c)$ ). The flood and ebb velocity profile profiles are then very different in the reach where salt is present on flood but not on ebb.

\section{Discussion}

The baroclinic residual force balance, salinity equation, and solutions considered here (equations (22) to (28)) bear a strong formal resemblance to those used in earlier investigations of the interaction of the time-average density and salinity fields by Rattray and Hansen [1962], Hansen and Rattray [1965], Hamilton and Rattray [1978], Officer [1976], Chatwin [1976], Oey [1984], and Prandle [1985]. Our application of these equations to the weakly stratified and partially mixed regimes differs, however, in several physically important ways from earlier treatments. First, arbitrary constants have been eliminated through the use of a barotropic tidal model. The horizontal tidal salinity dispersion term found in earlier models is explicitly represented by a tidal salinity-velocity correlation. vertical eddy diffusivity in the weakly stratified case is understood to represent turbulent mixing in a stratified boundary layer and has been specified using boundary layer methods. Vertical mixing in partially mixed estuaries is much weaker than in weakly stratified systems. The most probable mechanism in this case is random internal wave interactions, as specified by Gargett [1984], and Gargett and Holloway [1984]. Second, it is exactly this relation of vertical mixing to specific physical processes which allows the distinction between partially mixed and weakly stratified systems to be drawn. More detailed analysis of salt transport mechanisms [Jay, 1987] shows that baroclinic transport of water and salt is unimportant in the weakly stratified case. The salt in the weakly stratified state therefore acts, except for its role in modifying the vertical eddy diffusivity, as an essentially passive contaminant. So long as $R i_{g}$ remains subcritical, cal mixing is strong and the stratification and residual circulation arise almost entirely from barotropic nonlinearities. It is only when the stratification becomes strong enough to severely limit the penetration of the boundary 
layer turbulence into the flow that the baroclinic advection is important. This is the partially mixed case. The stronger the stratification, the weaker the mixing and the more important the role of baroclinic advection in driving a two-layer flow and in the salt balance [ Jay, 1987].

Third, the scaling indicates that vertical advection of the salinity field by the tidal and mean flows is relatively unimportant in both weakly stratified and partially mixed flows. ${ }^{0} W_{i}^{1}=0$ for all $\mathrm{x}$, and the only mean vertical velocity arises from the barotropic flow. If topography were to be represented in the model, ${ }^{0} W_{i}^{1}$ would certainly be nonzero, but the small vertical separation of inward and outward salt transports [Jay and Smith, 1989] argues against the importance of entrainment in the prototype. Placing the analysis of estuarine residual flow in its proper context as a branch of long-wave theory indicates the origin of this property - the ratio of vertical to horizontal velocities must be small because of the disparity in vertical and horizontal length scales. Finally, because the tidal flow is explicitly treated, it is possible to predict the tidal amplitude at which the spring-neap transition occurs.

Generation of residual flow in the weakly stratified case differs significantly from that in the two-layer case (part 1). First, bottom boundary-induced turbulence, rather than Kelvin-Helmholtz instabilities, is the primary cause of the vertical exchange. This minimizes the ebbflood asymmetry, so long as both flood and ebb currents are strong enough to allow robust mixing. Second, in the absence of $O(1)$ baroclinic wavelike motion in a weakly stratified flow, tidal-cycle advection of the isopycnals is dynamically unimportant because the density forcing is spatially and temporally nearly constant. Third, the internal mass transport that occurs in a two-layer flow is absent. In short, the major tidal-frequency mechanisms bringing about residual flow in the two-layer case are absent in the weakly stratified case. The result is a substantially weaker residual circulation and, relative to the highly stratified case, very little upstream bottom flow. The residual flow is essentially the outward river flow plus

a flow compensating for the inward Stokes drift.

With regard to the ebb-flood asymmetry, the harmonic decomposition employed in the weakly stratified case leads to a tidal flow in which the tidal velocity field is everywhere perfectly reversing. This means that the ebb-flood asymmetry is synonymous with the zero-frequency $O(\epsilon)$ residual circulation. In this respect, the theory agrees with the Hansen and Rattray [1965] model and other related theories. It agrees with the barotropic residual flow generation theories in that the convective nonlinearities are primarily responsible for generating this zerofrequency flow mode. The weakly stratified flow theory developed here is, furthermore, realistic in the sense that both the observed and predicted upstream bottom flow and residual shear are much smaller than in the two-layer case. It falls short as a description of reality, however, in that it does not fully account for the flood-ebb difference in boundary shear stress and thus in the velocity profiles arising from the influence of the mean flow. There are two possible remedies to this shortcoming. One is to incorporate an ebb-flood asymmetry directly into the tidal flow by determining separate ebb and flood eddy diffusivities iteratively. These would depend, in part, on the structure of the residual flow. The other is to begin with a vertically integrated model, akin to that used in part
1, and use it to determine the contribution of tidal cycle variations in bottom stress to the residual flow. This adds another forcing term to the residual momentum balance and would alter the salinity defect as well.

\section{Summary and Conclusions}

A perturbation method based on long-wave theory has been used herein to develop analytical models of flows in weakly stratified and partially mixed estuaries. The flows in these systems can be considered as the sum of weakly interacting barotropic and baroclinic modes. Only the barotropic mode contributes to the dominant tidal frequency flow, so the barotropic flow is nearly independent of the baroclinic flow. The barotropic flow is essentially that analyzed by Ianniello $[1977 a, b]$, in which the finite amplitude of the tidal wave drives an inward Stokes drift that is compensated by zero-frequency Eulerian flow. The internal vertical, buoyant restoring force is weak, and the baroclinic flow is degenerate in that an $O(1)$ baroclinic wave is absent. The $O\left(\epsilon_{i}\right)$ baroclinic flow is driven by the nearly uniform horizontal density gradient and resembles the "central regime" first described by Hansen and Rattray [1965].

The primary difference between weakly stratified and partially mixed estuaries concerns the dominant vertical exchange mechanism for salt and fresh water on the flood, the decisive part of the tidal cycle for the evolution of the residual flow. In weakly stratified flows, the shear is large enough relative to the stratification that bottom boundary-induced turbulent mixing may extend throughout most of the flow, being small only near the free surface, where the shear vanishes. In partially mixed estuaries, in contrast, strong turbulent mixing is confined by the stratification to a part of the flow near the bed; mixing in the interior is therefore weak even on flood. We hypothesize that the dominant mechanism producing this weak mixing is random internal wave mixing, but the exact identity of the mechanism is less important than its weakness. The present analysis differs from earlier studies of residual flow in two major respects. First, advection of salt by both the tidal and mean flows is treated directly. Second, this explicit representation of the tidal flow allows the arbitrary constants that have been used to describe horizontal and vertical transfer of mass and salt by the tidal circulation to be eliminated.

Two major factors distinguish the flows described herein from the highly stratified case analyzed in part 1. First, the tidal-frequency baroclinic flow is strong $(O(1))$ in highly stratified or salt wedge flows, but only $O(\epsilon)$ in weakly stratified and partially mixed estuaries. Second, because stratification damps bottom boundary-induced turbulence in the interfacial layer, the dominant mechanism causing the vertical exchange of water and salt in salt wedges is Kelvin-Helmholtz instabilities on the interface between the two layers. This mixing mechanism is unimportant in weakly stratified and partially mixed estuaries because of the absence of a strong, tidal-frequency internal oscillation to provide the requisite shear. Mixing is therefore weaker in salt wedges than in weakly stratified estuaries, but still stronger than in partially mixed systems.

A uniform channel model based on our analysis of the weakly stratified state successfully predicts residual velocity and salinity distributions in the Columbia River Estu- 
ary for low-flow spring tides. This model also correctly predicts the critical tidal amplitude at which the system returns to a highly stratified state after the spring tide. This transition occurs when stratification becomes strong enough that turbulent mixing can no longer be maintained in the interior. A similar model of the partially mixed condition shows that it can have only a transient existence in the Columbia River Estuary, because the river flow and tidal forcing are both too large. As soon as turbulent mixing is suppressed in the interior during periods of decreasing tidal forcing, the system again becomes highly stratified. Conversely, whenever internal mixing by KelvinHelmholtz instabilities destroys the two layer flow during periods of increasing tidal range, bottom boundaryinduced turbulent mixing can penetrate throughout the flow, allowing the establishment of a weakly stratified system. The model further shows that an estuary similar to the Columbia River Estuary, but with a smaller tidal range and river flow reduced by 2 orders of magnitude, would be partially mixed.

Finally, the generality of the analysis of estuarine residual flow presented herein and in part 1 has been enhanced by development of a classification system for narrow and shallow estuaries [ Jay and Smith, 1988]. This system uses two classification parameters. The first is a barotropic Froude number $F_{T}\left(=\epsilon_{b}\right)$. It is a measure of the tendency of the surface tide to generate residual flow via barotropic, finite amplitude wave mechanisms. These increase directly with $F_{T}$. The second is an internal Froude number $F_{B}$ based on the tidal excursion of the density field. It is equivalent to the expansion parameter for the baroclinic flow in part 1 and thus to the inverse of $\epsilon_{i}$ as defined herein. Maximum nonlinearity and, therefore, maximum generation of residual flow by nonlinearities associated with advection of the density field are achieved with an $F_{B}$ of 1 . This is also the value of $F_{B}$ that separates highly stratified estuaries from those that are weakly stratified or partially mixed. The distinction between partially mixed and weakly stratified estuaries is based on expression of the Richardson number $R i_{g}$ in (34c) as a relationship between $F_{T}$ and $F_{B}$.

Acknowledgments. This research was supported by NSF grants OCE-8208856, OCE-8504237, and OCE-8711790. Kathy Smith typed the equations and processed the text; Lin Sylwester drafted the figures.

\section{REFERENCES}

Abraham, G., Turbulence and mixing in stratified tidal flow, in Physical Processes in Estuaries, edited by J. Dronkers and W. van Leussen, pp 149-180, Springer-Verlag, New York, 1988.

Businger, J. A., J. C. Wyngaard, Y. Izumi, and E. F. Bradley, Flux profile relationships in the atmospheric surface layer, $J$. Atmos. Sci., 28, 190-201, 1971.

Chatwin, P. C., Some remarks on the maintenance of the salinity distribution in estuaries, Estuarine Coastal Mar. Sci., 4, 555$566,1976$.

Dyer, K. R., Estuaries, A Physical Introduction, pp. 9-12, John Wiley, New York, 1973.

Gargett, A. E., Vertical eddy diffusivity in the ocean interior, $J$. Mar. Res., 42, 359-393, 1984.

Gargett, A. E., and G. Holloway, Dissipation and diffusion by internal wave breaking, J. Mar. Res., 42, 15-27, 1984.

Hamilton, P., and M. Rattray, Jr., Theoretical aspects of estuarine circulation, in Estuarine Transport Processes, edited by B. Kjerfve, pp. 37-74, University of South Carolina Press, Columbia, 1978.
Hansen, D. V., and M. Rattray, Jr., Gravitational circulation in straits and estuaries, J. Mar. Res., 23, 104-122, 1965.

Hinze, J.O., Turbulence, 597 pp., McGraw-Hill, New York, 1959. Ianniello, J. P., Nonlinearly induced residual currents in tidally dominated estuaries, Ph.D. thesis, Univ. of Conn., Storrs, $1977 a$.

Ianniello, J. P., Tidally induced residual currents in estuaries of constant breadth and depth, J. Mar. Res., 35, 755-774, 1977b.

Ianniello, J. P., Tidally induced currents in estuaries of variable breadth and depth, J. Phys. Oceanogr., 9, 962-974, 1979.

Ianniello, J. P., Comments on tidally induced residual currents in estuaries: dynamics and near-bottom flow characteristics, $J$. Phys. Oceanogr., 11, 126-134, 1981.

Jay, D. A., Circulatory processes in the Columbia River Estuary, 169 pp., Columbia Riv. Est. Study Task., Astoria, Oreg., 1984. Jay, D. A., Residual circulation in shallow, stratified estuaries, Ph.D. thesis, Univ. of Wash., Seattle, 1987.

Jay, D. A., Estuarine salt and sediment transport: A Lagrangian approach, Estuarine Coastal Shelf Sci., in press, 1989.

Jay, D. A., and J. D. Smith, Residual circulation in, and classification of shallow estuaries, in Physical Processes in Estuaries, edited by J. Dronkers, and W. van Leussen, pp. 19-41. Springer-Verlag, New York, 1988,

Jay, D. A., and J. D. Smith, Circulation, density structure and neap-spring transitions in the Columbia River Estuary, Prog. Oceanogr., in press, 1989.

Jay, D. A., and J. D. Smith, Residual circulation in shallow estuaries: 1 . Highly stratified, narrow estuaries, J. Geophys. Res., this issue.

Long, C. E., A simple model for time-dependent stably stratified boundary layers, Department of Oceanography, Univ. of Wash., Seattle, Spec. Rep., 95, 170 pp. 1981.

Longuet-Higgins, M. S., On the transport of mass by time-varying ocean currents, Deep Sea Res., 16, 431-447, 1969.

Neal, V. T., Physical aspects of the Columbia River and its estuary, in The Columbia River Estuary and Adjacent Ocean Waters, edited by A. T. Pruter and D. L. Alverson, pp. 19-40, University of Washington Press, Seattle, 1972.

Oey, L.-Y., On steady salinity distribution and circulation in partially mixed and well mixed estuaries, J. Phys. Oceanogr., 14, 629-645, 1984.

Officer, C. B., Physical Oceanography of Estuaries (and Associated Coastal Waters), John Wiley, New York, 1976.

Perroud, P., The propagation of tidal waves into channels of gradually varying cross section, University of California College of Engineering Series 89(3), 1958.

Prandle, D., On salinity regimes and vertical structure of residual flows in narrow tidal estuaries, Estuarine Coastal Shelf Sci., 20, 615-635, 1985.

Pritchard, D. W., Observations of circulation in coastal plain estuaries. in Estuaries, Publ. 83 edited by G. H. Lauf, pp. 37-44, AAAS, Washington, D.C., 1967.

Rattray, M., Jr., and D. V. Hansen, A similarity solution for circulation in an estuary, J. Mar. Res., 20(2), 121-133, 1962.

Rattray, M., Jr., and E. Mitsuda, Theoretical analysis of conditions in a salt wedge, Estuarine Coastal Mar. Sci., 2, 375-394, 1974.

Sarabun, C. C., A. Brandt, M. A. Tyler, and H. H. Seliger, Breaking internal waves in Chesapeake Bay (abstract) Eos Trans. AGU, 65(45), 907, 1984.

Sherwood, C. R., D. A. Jay, R. B. Harvey, P. Hamilton, and C. D. Simenstad, Historical changes in the Columbia River Estuary, Prog. Oceanogr., in press, 1989.

Simpson, J. H., and J. R. Hunter, Fronts in the Irish Sea, Nature, $250,404-406,1974$.

Turner, J.S., Buoyancy Effects in Fluids, pp. 23-24, Cambridge University Press, New York, 1973.

Wieringa, J., A revaluation of the Kansas Mast influence on measurements of stress and cup anemometer overspeeding, Boundary Layer Meteorol., 18, 411-430, 1980.

D. A. Jay and J. D. Smith, Geophysics Program, AK-50, University of Washington, Seattle, WA 98195.

(Received April 13, 1988; accepted August 29, 1988.) 